TRANSACTIONS OF THE

AMERICAN MATHEMATICAL SOCIETY

Volume 358, Number 6, Pages 2507-2536

S 0002-9947(05)03747-5

Article electronically published on May 26, 2005

\title{
RESOLUTIONS FOR METRIZABLE COMPACTA IN EXTENSION THEORY
}

\author{
LEONARD R. RUBIN AND PHILIP J. SCHAPIRO
}

\begin{abstract}
We prove a $K$-resolution theorem for simply connected CWcomplexes $K$ in extension theory in the class of metrizable compacta $X$. This means that if $K$ is a connected CW-complex, $G$ is an abelian group, $n \in \mathbb{N}_{\geq 2}$, $G=\pi_{n}(K), \pi_{k}(K)=0$ for $0 \leq k<n$, and extdim $X \leq K$ (in the sense of extension theory, that is, $K$ is an absolute extensor for $X$ ), then there exists a metrizable compactum $Z$ and a surjective map $\pi: Z \rightarrow X$ such that:

(a) $\pi$ is $G$-acyclic,

(b) $\operatorname{dim} Z \leq n+1$, and

(c) $\operatorname{ext\operatorname {dim}Z} \leq K$.

This implies the $G$-resolution theorem for arbitrary abelian groups $G$ for cohomological dimension $\operatorname{dim}_{G} X \leq n$ when $n \in \mathbb{N}_{\geq 2}$. Thus, in case $K$ is an Eilenberg-Mac Lane complex of type $K(G, n)$, then (c) becomes $\operatorname{dim}_{G} Z \leq n$.

If in addition $\pi_{n+1}(K)=0$, then (a) can be replaced by the stronger statement,

(aa) $\pi$ is $K$-acyclic.

To say that a map $\pi$ is $K$-acyclic means that for each $x \in X$, every map of the fiber $\pi^{-1}(x)$ to $K$ is nullhomotopic.
\end{abstract}

\section{INTRODUCTION}

The Edwards-Walsh resolution theorem, the first resolution theorem for cohomological dimension, was proved in $\mathrm{Wa}$ (see also $\mathrm{Ed}]$ ). It states that if $X$ is a metrizable compactum and $\operatorname{dim}_{\mathbb{Z}} X \leq n(n \geq 0)$, then there exists a metrizable compactum $Z$ with $\operatorname{dim} Z \leq n$ and a surjective cell-like map $\pi: Z \rightarrow X$. This result, in conjunction with Dranishnikov's work ([Dr1] showing that in the class of metrizable compacta, $\operatorname{dim}_{\mathbb{Z}}$ is distinct from dim, was a key ingredient for proving that cell-like maps could raise dimension (see Ru1 for background). For the reader seeking fundamentals on the theory of cohomological dimension, $\operatorname{dim}_{G}$, the references [Ku], [Dr3], $\mathrm{Dy}$, and [Sh] could be helpful.

In $\mathrm{Wa}$ the author made use of the fact that cohomological dimension with respect to the abelian group $\mathbb{Z}$ could be defined in terms of extensions of maps. That is, $\operatorname{dim}_{\mathbb{Z}} X \leq n$ if and only if every map of a closed subspace of $X$ to (a CWcomplex of type) $K(\mathbb{Z}, n)$ can be extended to a map of $X$ to $K(\mathbb{Z}, n)$. Indeed (see Theorem 26 of [Ku] ), for any abelian group $G, \operatorname{dim}_{G} X \leq n$ if and only if every map

Received by the editors March 13, 2002 and, in revised form, May 11, 2004.

2000 Mathematics Subject Classification. Primary 55P55, 54F45.

Key words and phrases. Bockstein basis, Bockstein inequalities, Čech cohomology, cell-like map, cohomological dimension, CW-complex, dimension, Edwards-Walsh resolution, EilenbergMac Lane complex, $G$-acyclic resolution, inverse sequence, $K$-acyclic resolution, Moore space, shape of a point, simplicial complex. 
of a closed subspace of $X$ to $K(G, n)$ can be extended to a map of $X$ to $K(G, n)$. Always by $K(G, n)$ we mean an Eilenberg-Mac Lane CW-complex of type $K(G, n)$, and such is characterized (up to homotopy equivalence) by having $\pi_{n}=G$ and $\pi_{k}$ trivial for all other $k$.

Motivated in part by this, a concept called "extension theory" was introduced by A. Dranishnikov (see DD for some background on this notion). For a CWcomplex $K$ and a space $X$, one writes extdim $X \leq K$ (variously, $X \tau K, K \in \operatorname{AE}(X)$, $\operatorname{dim} X \leq K)$ if for each closed subspace $A$ of $X$ and map $f: A \rightarrow K$, there exists a map $F: X \rightarrow K$ such that $F \mid A=f$. If $K=K(G, n)$, then $\operatorname{extdim} X \leq K$ is equivalent to $\operatorname{dim}_{G} X \leq n$.

Now a map is cell-like provided that each of its fibers is cell-like, or, equivalently, has the shape of a point (MS1]). Every cell-like compactum has trivial reduced Čech cohomology with respect to any abelian group $G$. This means that for every abelian group $G$, every cell-like map is $G$-acyclic, i.e., all its fibers have trivial reduced Cech cohomology with respect to the group $G$. This is equivalent to the statement that every map of such a fiber to $K(G, n)$ is nullhomotopic.

The latter notion may be generalized as follows. For a given $\mathrm{CW}$-complex $K$, a metrizable compactum $X$ is called $K$-acyclic if every map of it to $K$ is nullhomotopic. Moreover, one should recall that when a Hausdorff compactum or metrizable space $X$ has $\operatorname{dim} X \leq n$, then also $\operatorname{dim}_{\mathbb{Z}} X \leq n$.

With these ideas in mind, one may ask, what kind of parallel resolution theorems can be obtained under the assumption that $\operatorname{extdim} X \leq K$, where $K$ is a CWcomplex different from $K(\mathbb{Z}, n)$ ? It turns out that it is not possible to always have cell-like resolutions as in the Edwards-Walsh theorem, nor can one even require in such propositions that $\operatorname{dim} Z \leq n$ be true (see [KY2]). So, what kind of resolution theorems can we expect? The main result of this paper is:

1.1. Theorem. Let $K$ be a connected $C W$-complex, $G$ an abelian group, $n \in \mathbb{N}_{\geq 2}$, $G=\pi_{n}(K)$, and $\pi_{k}(K)=0$ for $0 \leq k<n$. Then for each metrizable compactum $X$ with $\operatorname{extdim} X \leq K$, there exists a metrizable compactum $Z$ and a surjective map $\pi: Z \rightarrow X$ such that:

(a) $\pi$ is G-acyclic,

(b) $\operatorname{dim} Z \leq n+1$, and

(c) $\operatorname{extdim} Z \leq K$.

If in addition, $\pi_{n+1}(K)=0$, then we may replace (a) by the stronger statement:

(aa) $\pi$ is $K$-acyclic.

As a corollary to Theorem 1.1, we get the $G$-acyclic resolution theorem in cohomological dimension theory.

1.2. Corollary. Let $G$ be an abelian group and let $X$ be a metrizable compactum with $\operatorname{dim}_{G} X \leq n, n \in \mathbb{N}_{\geq 2}$. Then there exists a metrizable compactum $Z$ and a surjective map $\pi: Z \rightarrow X$ such that:

(a) $\pi$ is $G$-acyclic,

(b) $\operatorname{dim} Z \leq n+1$, and

(c) $\operatorname{dim}_{G} Z \leq n$.

In [Le one finds another approach to Corollary 1.2. We mention that the Edwards-Walsh theorem has been generalized to the class of arbitrary metrizable spaces by Rubin and Schapiro ([RS] $)$ and to the class of arbitrary compact Hausdorff 
spaces by Mardešić and Rubin ([MR]). Corollary 1.2 was proved by Dranishnikov ([Dr2]) for the group $G=\mathbb{Z} / p$, where $p$ is an arbitrary prime number, but with the stronger outcome that $\operatorname{dim} Z \leq n$. Later, Koyama and Yokoi ([KY1]) were able to obtain this $\mathbb{Z} / p$-resolution theorem of Dranishnikov both for the class of metrizable spaces and for that of compact Hausdorff spaces.

In their work [KY2], Koyama and Yokoi made a substantial amount of progress in the resolution theory of metrizable compacta, that is, towards proving Corollary 1.2. Their method relies heavily on the existence of Edwards-Walsh resolutions, which had been studied by Dydak and Walsh in [DW], and which had been applied originally, in a rudimentary form, in Wa. The definition of an Edwards-Walsh resolution can be found in KY2, but we shall not use it herein.

To overcome a flaw in the proof of Lemma 4.4 of [DW], Koyama and Yokoi proved the existence of Edwards-Walsh resolutions for some groups $G$, but under a stronger set of assumptions on $G$ than had been thought necessary in [DW]. It is still not known if these stronger assumptions are needed to insure the existence of the resolutions. Nevertheless, Koyama and Yokoi proved substantial $G$-acyclic resolution theorems. Let us state two of the important theorems from KY2 (Theorems 4.9 and 4.12 , respectively), which greatly influenced the direction of the work in this paper.

1.3. Theorem. Corollary 1.2 is true for every torsion free abelian group $G$.

1.4. Theorem. Let $G$ be an arbitrary abelian group and let $X$ be a metrizable compactum with $\operatorname{dim}_{G} X \leq n, n \in \mathbb{N}_{\geq 2}$. Then there exists a surjective $G$-acyclic map $\pi: Z \rightarrow X$ from a metrizable compactum $Z$, where $\operatorname{dim} Z \leq n+2$ and $\operatorname{dim}_{G} Z \leq n+1$.

In case $G$ is a torsion group, Koyama and Yokoi prove (Theorem 4.11 in [KY2]) that Corollary 1.2 holds, but without part (c). Of course Theorem 1.4 falls short of Corollary 1.2. We observed that one of the main reasons for the relative weakness of this theorem was that Koyama and Yokoi proved it by an indirect technique, a type of "finesse." Their approach depends heavily on the Bockstein basis theorem and the Bockstein inequalities (see $[\mathrm{Ku}]$ ), instead of the more direct method, involving Edwards-Walsh resolutions, used to prove Theorem 1.3.

We want to point out that Theorem 1.3 includes as a corollary, and therefore redeems, the $\mathbb{Q}$-resolution theorem of Dranishnikov ([Dr5]-but see also [Dr6], where a different proof is given). The Koyama and Yokoi proof shows that in the proof of Theorem 3.2 of [Dr5], the statement that $\alpha_{m} \circ \omega_{m}$ is an Edwards-Walsh resolution over $\tau_{m}^{(n+1)}$ is not true. This was a subtle point; to fully understand it, the interested reader may examine the text immediately following the proof of Fact 1 of the proof of Theorem 3.1 in KY2. Getting around the barrier naturally led to a quite complicated construction.

Our proof of Theorem 1.1 will be direct, using extensions which are different from Edwards-Walsh resolutions. But we will use a type of pseudo-Bockstein basis denoted $\sigma_{0}(G)$ (section 4 ). This will allow us to deal with the groups $\mathbb{Z} / p^{\infty}$ as well as the other groups involved. We shall employ the technique of inverse sequences both to represent our given space $X$ and to determine the resolving space $Z$. The map $\pi: Z \rightarrow X$ will be obtained in a standard, yet complicated manner similar to that used in $[\mathrm{Wa}$. 
An outline of the sections of this paper goes as follows.

1. Introduction, including some historical facts about acyclic resolutions with respect to certain groups.

2. Eilenberg-Mac Lane complexes $K(G, n)$ and direct sequences of $K(G, n)$ 's with an application to $K\left(\mathbb{Z} / p^{\infty}, n\right)$.

3. Some theory of inverse sequences of compacta along with Dranishnikov's lemma for obtaining maps of a compactum to a given compactum with discernable fibers.

4. Some basic algebra needed for this work including a variant Bockstein theory.

5. Extensions of generic type will be defined here. The concept involves starting with a simplicial complex of dimension $\leq n+1$, removing the interiors of all its $(n+1)$-simplexes, replacing them with other objects to create a $\mathrm{CW}$-complex, and then defining a map of this $\mathrm{CW}$-complex back to the polyhedron of the original simplicial complex.

6. Layered extensions, certain CW-complexes, are introduced in this section. Using the concepts from section 5 , but with a more specific goal in mind, we build extensions in a finite number of layers and then in a reverse process attach $(n+1)$-cells to improve the homotopy groups of special subspaces of these $\mathrm{CW}$-complexes.

7. Our proof of the $G$-acyclic resolution theorem involves a certain recursive process. The purpose of this section is to show how to make the move in the induction from stage $w$ to stage $w+1$.

8. Pulling together the elements of the preceding sections, this ultimate one will provide the final steps in our proof of Theorem 1.1.

The authors express their gratitude to Professor Ivan Ivanšić of the University of Zagreb for his many helpful discussions with us during the preparation of this manuscript. Our thanks go to M. Levin who indicated to us that our results could be sharpened from those originally proposed. We are extremely grateful for a long and studied report from the referee.

\section{Lemmas for EILENBERG-Mac LANe COMPleXes}

The purpose of this section is to illuminate certain facts about EilenbergMac Lane complexes in a form that will be useful for us. Map will mean continuous function, and for a simplicial complex $K$, its polyhedron $|K|$ will always be given the weak topology based on the triangulation $K$.

2.1. Lemma. Suppose that $\left(G_{i}, p_{i+1}^{i}\right)$ is a direct sequence of injective homomorphisms $p_{i+1}^{i}: G_{i} \rightarrow G_{i+1}$ of abelian groups whose limit is the abelian group $G$. Then for each $n \in \mathbb{N}$, there exists a direct sequence $\left(K\left(G_{i}, n\right), q_{i+1}^{i}\right)$ of $K\left(G_{i}, n\right)$ 's and embeddings $q_{i+1}^{i}: K\left(G_{i}, n\right) \rightarrow K\left(G_{i+1}, n\right)$, which induce injective homomorphisms on homotopy groups, and whose limit equals $K(G, n)$.

Proof. Our proof is only for $n \geq 2$; we leave the case $n=1$ to the reader. Suppose $m \in \mathbb{N}$ and we have constructed CW-complexes $K_{1}, \ldots, K_{m}$, and maps $q_{i+1}^{i}: K_{i} \rightarrow$ $K_{i+1}$ for $i<m$ such that for $1 \leq i \leq m$,

(1) $K_{i}$ is a $K\left(G_{i}, n\right)$, and

(2) $\pi_{n}\left(q_{i+1}^{i}\right): \pi_{n}\left(K_{i}\right) \rightarrow \pi_{n}\left(K_{i+1}\right)$ is injective for $i<m$. 
Let $S$ be a bouquet of $\alpha$ copies of $S^{n}$, where $\alpha$ is the cardinality of a generating set of $G_{m+1}$. Form the wedge $K_{m} \vee S$ and note that $\pi_{j}\left(K_{m} \vee S\right)=0$ for $j<n$. Also note that by Theorem V.3.1 of [ $\mathrm{Hu}], \pi_{n}\left(K_{m} \vee S\right)$ is isomorphic to $G_{m} \oplus \pi_{n}(S) \oplus H$. Attach as needed a set of $(n+1)$-cells to $K_{m} \vee S$ to kill the summand $H$. This yields a CW-complex $L_{1}$ with $\pi_{j}\left(L_{1}\right)=0$ for $j<n$ and $\pi_{n}\left(L_{1}\right) \approx G_{m} \oplus \pi_{n}(S)$. Next attach $(n+1)$-cells to $L_{1}$ to form a CW-complex $L_{2}$ with $\pi_{n}\left(L_{2}\right) \approx G_{m} \oplus G_{m+1}$ and $\pi_{j}\left(L_{2}\right)=0$ for $j<n$.

Fix a set $U$ of generators of $G_{m}$. For each $u \in U$, we consider the element $u-p_{m+1}^{m}(u) \in \pi_{n}\left(L_{2}\right)$, and attach an $(n+1)$-cell to $L_{2}$ to kill this element. The resulting CW-complex $L_{3}$ has the property that $\pi_{j}\left(L_{3}\right)=0$ for $j<n$ and $\pi_{n}\left(L_{3}\right) \approx$ $G_{m+1}$. Moreover, the homomorphism on $\pi_{n}$ induced by the inclusion $K_{m} \hookrightarrow L_{3}$ injects $G_{m}$ into $G_{m+1}$ as a homomorphism equivalent to $p_{m+1}^{m}: G_{m} \rightarrow G_{m+1}$.

The final step in producing $K_{m+1}$ is to attach cells of dimensions $n+2$ and higher to $L_{3}$ as necessary to kill all the higher homotopy groups. From this construction it is clear that $K_{m+1}$ is a $K\left(G_{m+1}, n\right)$ and that the inclusion $q_{m+1}^{m}: K_{m} \rightarrow K_{m+1}$ induces the injective homomorphism $p_{m+1}^{m}: G_{m} \rightarrow G_{m+1}$. This concludes our proof.

We shall apply Lemma 2.1 in the sequel for $p \in \mathbb{P}$ and $G=\mathbb{Z} / p^{\infty}$. Recall that $\mathbb{Z} / p^{\infty}$ may be represented as the direct limit of a direct sequence $\left(G_{i}, p_{i+1}^{i}\right)$, where for each $i \in \mathbb{N}$,

(1) $G_{i}=\mathbb{Z} / p^{i}$, and

(2) $p_{i+1}^{i}: G_{i} \rightarrow G_{i+1}$ is the injective homomorphism induced by multiplication by $p$.

The next could be used to prove the Bockstein inequality, $\operatorname{dim}_{\mathbb{Z}_{p} \infty} X \leq \operatorname{dim}_{\mathbb{Z} / p} X$.

2.2. Corollary. For each $p \in \mathbb{P}$ and $n \in \mathbb{N}$, there exists a direct sequence $\left(K\left(\mathbb{Z} / p^{i}, n\right), q_{i+1}^{i}\right)$ of embeddings $q_{i+1}^{i}$ which, on $n$-th homotopy groups, induce the injective homomorphisms in (2), and such that the limit of the sequence equals $K\left(\mathbb{Z} / p^{\infty}, n\right)$.

In Exercise 5 of [Wa], it is stated that $K\left(\bigoplus_{1}^{l} \mathbb{Z}, n\right)$ is an Eilenberg-Mac Lane space of type $\prod_{1}^{l} K(\mathbb{Z}, n)$ for any $l \in \mathbb{N}$. A similar result is used implicitly in $[\mathrm{KY} 2$ and elsewhere for groups $G$ different from $\mathbb{Z}$. On the other hand, as pointed out on page 401 of $[\mathrm{Sp}$ (in reference to a result of C. H. Dowker), the product of two CW-complexes need not be a CW-complex, although it will be if at least one of the factors is locally compact. This brings up the question: what is really needed concerning $K\left(\bigoplus_{1}^{l} G, n\right)$ in the theory of resolutions in cohomological dimension? Let us give an answer which applies to a large class of spaces.

If $K$ and $L$ are $\mathrm{CW}$-complexes, then by $K \times_{\mathrm{CW}} L$ is meant the set $K \times L$ with the weak topology induced by the $\mathrm{CW}$-structure obtained from the product of the cells of $K$ and $L$. This topology need not agree with the product topology, but the identity function $i: K \times{ }_{\mathrm{CW}} L \rightarrow K \times L$ is continuous. Recall that a $k$-space is a topological space whose topology is compactly generated. Polyhedra with the weak topology, CW-complexes, first countable spaces, and compact Hausdorff spaces are examples of $k$-spaces. 
Let us state Proposition 1.5 of [DD] here for the reader's convenience.

2.3. Proposition. Let $X$ be a $k$-space and let $K, L$ be $C W$-complexes. Then a function $f: X \rightarrow K \times{ }_{\mathrm{CW}} L$ is continuous if and only if $i \circ f: X \rightarrow K \times L$ is continuous.

2.4. Lemma. Let $H=\bigoplus_{1}^{l} G$ be a finite direct sum of copies of an abelian group $G$, let $X$ be a paracompact $k$-space, and let $n \in \mathbb{N}$. Then $\operatorname{dim}_{G} X \leq n$ if and only if for every closed subset $A$ of $X$ and map $f: A \rightarrow K(H, n)$, there exists a map $F: X \rightarrow K(H, n)$ which is an extension of $f$.

Proof. Using Proposition 2.3, it is not difficult to see that $\pi_{k}\left(\prod_{1 \mathrm{CW}}^{l} K(G, n)\right) \approx$ $\pi_{k}\left(\prod_{1}^{l} K(G, n)\right)$ for all $k$. Hence $\prod_{1}^{l} K(G, n)$ is a $K(H, n)$.

If $\operatorname{dim}_{G} X \leq n$, then surely $\prod_{1}^{l} K(G, n)$ is an absolute extensor for $X$, since the same is true for each of its factors. Applying Proposition 2.3 again, one concludes that for any closed subset $A$ of $X$ and map $f: A \rightarrow K(H, n)$, there is a map $F: X \rightarrow K(H, n)$ extending $f$.

For the converse, let $A \subset X$ be closed and $f: A \rightarrow K(G, n)$ be a map. Denote by $f^{*}: A \rightarrow \prod_{1 \mathrm{CW}}^{l} K(G, n)=K(H, n)$ the function given by $f^{*}(a)=(f(a), \ldots, f(a))$. Using Proposition 2.3, we see that $f^{*}$ is continuous, so by assumption it extends to a map $F^{*}: X \rightarrow \prod_{1 \mathrm{CW}}^{l} K(G, n)$. Now Proposition 2.3 shows that $i \circ F^{*}: X \rightarrow$ $\prod_{1}^{l} K(G, n)$ is continuous. The map $F=p_{1} \circ i \circ F^{*}: X \rightarrow K(G, n)$, where $p_{1}$ is projection to the first coordinate, is an extension of $f$.

2.5. Lemma. Let $H=\bigoplus_{1}^{l} G$ be a finite direct sum of copies of an abelian group $G$, let $X$ be a metrizable space, and let $n \in \mathbb{N}$. Assume that $\operatorname{dim}_{G} X \leq n, \operatorname{dim} X \leq$ $n+1$, and $K$ is a $C W$-complex having the property that

$$
\pi_{k}(K) \approx \begin{cases}0 & \text { if } k<n, \\ H & \text { if } k=n .\end{cases}
$$

Then $\operatorname{extdim} X \leq K$.

Proof. Surely, $K \subset K(H, n)$, where $K(H, n) \backslash K$ consists of cells of dimensions $k$, $k \geq n+2$. If $A \subset X$ and $f: A \rightarrow K$ is a map, then by Lemma 2.4 there exists a map $F: X \rightarrow K(H, n)$ extending $f$. Dimensional considerations and the homotopy extension theorem show that we may assume that $F(X) \subset K(H, n)^{(n+1)}=K$.

\section{LEMMAS FOR INVERSE SEQUENCES}

Our proof of the main result will require certain manipulations of inverse sequences of metric compacta. This section will contain the needed results. 
The next lemma follows from Corollary 1 of [MS2].

3.1. Lemma. Let $\mathbf{X}=\left(X_{i}, p_{i}^{i+1}\right)$ be an inverse sequence of metric compacta $\left(X_{i}, d_{i}\right)$. Then there exists a sequence $\left(\delta_{i}\right)$ of positive numbers such that if $\mathbf{Y}=$ $\left(X_{i}, q_{i}^{i+1}\right)$ is an inverse sequence and $d_{i}\left(p_{i}^{i+1}, q_{i}^{i+1}\right)<\delta_{i}$ for each $i$, then $\lim \mathbf{Y}=$ $\lim \mathbf{X}$.

We shall call such $\left(\delta_{i}\right)$ a sequence of stability for $\mathbf{X}$.

Henceforth if we write that $f:|L| \rightarrow|K|$ is a simplicial map, we mean that $f$ is induced by a simplicial function $L \rightarrow K$. As usual, when $f: L \rightarrow K$ is a simplicial map of simplicial complexes, we shall use $f:|L| \rightarrow|K|$ to denote the induced map of polyhedra. Let us recall some terminology from section 2 of [JR]. Let $K$ be a finite simplicial complex, let $X$ be a space, and let $f: X \rightarrow|K|$ be a map. Then a map $g: X \rightarrow|K|$ is called a $K$-modification of $f$ if whenever $x \in X$ and $f(x) \in \sigma \in K$, then $g(x) \in \sigma$. It should be observed that if $L$ is a simplicial complex, $X=|L|$, and $g:|L| \rightarrow|K|$ is a simplicial approximation to $f$, then $g$ is a $K$-modification of $f$. One calls $f$ a $K$-irreducible map if each $K$-modification $g$ of $f$ is surjective. Note that in this case, $f$ is surjective and for any subdivision $M$ of $K, f$ is $M$-irreducible.

From Theorem 3.11 of $\mathrm{JR}$ ] we may deduce the following.

3.2. Lemma. Let $X$ be a metrizable compactum. Then we may write $X$ as the limit of an inverse sequence $\mathbf{Q}=\left(\left|Q_{i}\right|, q_{i}^{i+1}\right)$ of compact metric polyhedra, where each bonding map $q_{i}^{i+1}$ is a $Q_{i}$-irreducible surjection. If $\operatorname{card} X \geq 2$, then we may assume that card $Q_{i} \geq 2$ for each $i \in \mathbb{N}$.

Let us recall that by an interior cover of a space $X$ we mean a cover of $X$ by sets whose interiors also cover $X$. We need Dranishnikov's definition of a certain type of Lebesgue number.

Let $X$ be a compact metric space and let $\mathcal{M}$ be an interior cover of $X$ by closed sets such that no member of $\mathcal{M}$ equals $X$. Then there is a positive number $\lambda^{u}(\mathcal{M})$ given by

$$
\lambda^{u}(\mathcal{M})=\max \{r \mid \forall x \in X \exists M \in \mathcal{M}, B(x, r) \subset M\} .
$$

The next lemma is clearly true.

3.3. Lemma. If $X$ is a nonempty compact metric space and $\mathcal{M}$ is an interior cover of $X$ by closed sets such that no member of $\mathcal{M}$ equals $X$, then $\lambda^{u}(\mathcal{M}) / 4<$ $\lambda^{u}(\mathcal{M}) \leq \operatorname{mesh} \mathcal{M}$.

For a given simplicial complex $K$, let $\overline{s t} K$ denote the set of closed vertex stars of $K$. Surely $\overline{\text { st }} K$ is an interior cover of $|K|$ by closed sets.

3.4. Lemma. Let $X$ be a metrizable compactum with $\operatorname{card} X \geq 2$. Then there exists an inverse sequence $\mathbf{K}=\left(\left|K_{i}\right|, p_{i}^{i+1}\right)$ of compact metric polyhedra $\left(\left|K_{i}\right|, d_{i}\right)$ along with a sequence $\left(\delta_{i}\right)$ of stability for $\mathbf{K}$ such that $\lim \mathbf{K}=X$, and for each $i \in \mathbb{N}$,

(a) $\operatorname{mesh} K_{i}<\delta_{i}$,

(b) no member of $\overline{\mathrm{st}} K_{i}$ equals $\left|K_{i}\right|$, and

(c) $\operatorname{mesh}\left(\overline{\mathrm{st}} K_{i+1}\right)<\lambda^{u}\left(\overline{\mathrm{st}} K_{i}\right) / 4$.

We may also specify that for some $m \in \mathbb{N}$, whenever $i \geq m$,

(d) $p_{i}^{i+1}:\left|K_{i+1}\right| \rightarrow\left|K_{i}\right|$ is a $K_{i}$-irreducible simplicial map. 
Proof. Write $X=\lim \mathbf{Q}$ of compact metric polyhedra $\left(Q_{i}, d_{i}\right)$ with card $Q_{i} \geq 2$ for each $i \in \mathbb{N}$ as in Lemma 3.2, and let $\left(\rho_{i}\right)$ be a sequence of stability for $\mathbf{Q}$. For each $i$, put $\delta_{i}=\rho_{i} / 2$.

Below when we select a subdivision $K_{i}$ of $Q_{i}$, we shall tacitly (without loss of generality since card $Q_{i} \geq 2$ for each $i \in \mathbb{N}$ ) assume that no element of $\overline{\operatorname{st}} K_{i}$ equals $\left|K_{i}\right|$. Let $K_{1}$ be a subdivision of $Q_{1}$ with mesh $K_{1}<\delta_{1}$. Suppose that $i \in \mathbb{N}$ and for each $1 \leq j \leq i$, we have chosen a subdivision $K_{j}$ of $Q_{j}$ with mesh $K_{j}<\delta_{j}$ and, when $1<j$, a map $p_{j-1}^{j}:\left|K_{j}\right| \rightarrow\left|K_{j-1}\right|$ which is a simplicial approximation to $q_{j-1}^{j}$. Assume moreover that when $j<i, \operatorname{mesh}\left(\overline{\mathrm{st}} K_{j+1}\right)<\lambda^{u}\left(\overline{\mathrm{st}} K_{j}\right) / 4$. Then select a subdivision $K_{i+1}$ of $Q_{i+1}$ with mesh $K_{i+1}<\delta_{i+1}$, mesh $\left(\overline{\operatorname{st}} K_{i+1}\right)<\lambda^{u}\left(\overline{\operatorname{st}} K_{i}\right) / 4$, and which supports a simplicial approximation $p_{i}^{i+1}:\left|K_{i+1}\right| \rightarrow\left|K_{i}\right|$ of $q_{i}^{i+1}$.

Note that $d_{i}\left(q_{i}^{i+1}, p_{i}^{i+1}\right)<\delta_{i}$. Then it is easy to check that with $\mathbf{K}=\left(\left|K_{i}\right|, p_{i}^{i+1}\right)$ and $m=1$, we have satisfied all the requirements.

3.5. Lemma. When an inverse sequence $\mathbf{K}$ of metric polyhedra $\left(\left|K_{i}\right|, d_{i}\right)$ satisfies (c) of Lemma 3.4, then for each $i<j$,

$$
\operatorname{mesh}\left(\overline{\operatorname{st}} K_{j}\right)<\lambda^{u}\left(\overline{\operatorname{st}} K_{i}\right) / 4 .
$$

Proof. Just apply Lemma 3.3 recursively.

3.6. Definition. Whenever $X$ is a metrizable compactum, then we shall refer to an inverse sequence $\mathbf{K}$ of metric polyhedra $\left(\left|K_{i}\right|, d_{i}\right)$ which admits a sequence $\left(\delta_{i}\right)$ of positive numbers and $m \in \mathbb{N}$ so that (a)-(d) of Lemma 3.4 are satisfied as a representation of $X$ which is stable and simplicially irreducible from index $m$.

Of course Lemma 3.4 and its proof show that,

3.7. Lemma. Every metrizable compactum $X$ with $\operatorname{card} X \geq 2$ has a representation $\mathbf{K}$ which is stable and simplicially irreducible from index $m=1$.

We want next to define a certain type of move which when applied to such $\mathbf{K}=\mathbf{K}_{0}$ as in Definition 3.6 results in a $\mathbf{K}_{1}$ which is also a stable and simplicially irreducible (from some index $m$ ) representation of $X$. We will then show that if this procedure is repeated recursively in a controlled manner, resulting in a sequence $\mathbf{K}_{1}, \mathbf{K}_{2}, \ldots$, then there will be a limit $\mathbf{K}_{\infty}=\lim _{j \rightarrow \infty}\left(\mathbf{K}_{j}\right)$ which also will be a representation of $X$.

3.8. Definition. Let $\left(\epsilon_{i}\right)$ be a sequence of positive numbers, let $X$ be a metrizable compactum, and let $\mathbf{K}=\left(\left|K_{i}\right|, p_{i}^{i+1}\right)$ be a representation of $X$ which is stable and simplicially irreducible from index $m_{1}$ with $\left(\delta_{i}\right)$ an associated sequence of stability. Let $m \in \mathbb{N}_{\geq m_{1}}$ and define $\delta_{i}^{\prime}=\delta_{i}$ if $1 \leq i<m, \delta_{m}^{\prime}=\frac{1}{2}\left[\delta_{m}-\operatorname{mesh} K_{m}\right]$, and $\delta_{i}^{\prime}=\delta_{i} / 2$ if $i>m$. There exists a sequence $\Sigma=L_{m}, L_{m+1}, L_{m+2}, \ldots$ such that for each $i \geq m$,

(a) $L_{i}$ is a subdivision of $K_{i}$ with mesh $L_{i}<\min \left\{\varepsilon_{i}, \delta_{i}^{\prime}\right\}$, and

(b) if $i \geq m+1$ there is a simplicial approximation $l_{i-1}^{i}:\left|L_{i}\right| \rightarrow\left|L_{i-1}\right|$ to the $\operatorname{map} p_{i-1}^{i}$.

Create a new inverse sequence $\mathbf{L}=\left(\left|L_{i}\right|, l_{i}^{i+1}\right)$ by setting, in addition to what we have just described, $L_{i}=K_{i}$ and $l_{i}^{i+1}=p_{i}^{i+1}$ in case $1 \leq i<m$. Observe that $d_{m}\left(p_{m}^{m+1}, l_{m}^{m+1}\right) \leq \operatorname{mesh} \Sigma<\frac{1}{2}\left[\delta_{m}-\operatorname{mesh} K_{m}\right]=\delta_{m}^{\prime}$. Hence if $g:\left|L_{m+1}\right| \rightarrow\left|L_{m}\right|$ 
is a map and $d_{m}\left(g, l_{m}^{m+1}\right)<\delta_{m}^{\prime}$, we may conclude that $d_{m}\left(g, p_{m}^{m+1}\right)<\delta_{m}$. Indeed, and we leave it to the reader to check, the following is true:

(c) for each $i$, if $g:\left|L_{i+1}\right| \rightarrow\left|L_{i}\right|$ is a map and $d_{i}\left(g, l_{i}^{i+1}\right)<\delta_{i}^{\prime}$, then $d_{i}\left(g, p_{i}^{i+1}\right)<$ $\delta_{i}$

By exercising some additional care in the construction of $\mathbf{L}$, we may guarantee that (c) of Lemma 3.4 applies to it, and

(d) for all $i, d_{i}\left(p_{i}^{i+1}, l_{i}^{i+1}\right)<\epsilon_{i}$ and, of course, $p_{i}^{i+1}=l_{i}^{i+1}$ if $i<m$.

We shall call such $\left(\mathbf{L},\left(\delta_{i}^{\prime}\right)\right)$ an $m$-shift of $\left(\mathbf{K},\left(\delta_{i}\right)\right)$ from $\Sigma$.

It is routine to check that the next lemma holds true.

3.9. Lemma. Let $X$ be a metrizable compactum and let $\mathbf{K}_{0}$ be a stably irreducible representation of $X$ with $\left(\delta_{i}^{0}\right)$ a sequence of stability and which is simplicial from index $m_{1}$. For every $m_{1}$-shift $\left(\mathbf{K}_{1},\left(\delta_{i}^{1}\right)\right)$ of $\left(\mathbf{K}_{0},\left(\delta_{i}^{0}\right)\right)$ from $\Sigma_{1}$ (an appropriate subdivision of the triangulation of the $m_{1}$-term of $\left.\mathbf{K}_{0}\right), \mathbf{K}_{1}$ is a representation of $X$ which is stable and simplicially irreducible from index $m_{1}$ with $\left(\delta_{i}^{1}\right)$ an associated sequence of stability. It satisfies (c) of Definition 3.8 with $\left(\delta_{i}^{\prime}\right)=\left(\delta_{i}^{1}\right)$ and $\left(\delta_{i}\right)=$ $\left(\delta_{i}^{0}\right)$. The terms (as metric spaces) in $\mathbf{K}_{0}$ and $\mathbf{K}_{1}$ are equal. For $i<m_{1}, \delta_{i}^{0}=\delta_{i}^{1}$, the terms with index $i$ have the same triangulations in $\mathbf{K}_{0}$ and $\mathbf{K}_{1}$, and the bonding maps in $\mathbf{K}_{0}$ and $\mathbf{K}_{1}$ with subscript $i$ are equal. For $i \geq m_{1}$, $\delta_{i}^{1}$ need not equal $\delta_{i}^{0}$, the triangulation of the term in $\mathbf{K}_{1}$ with index $i$ is a subdivision of that in $\mathbf{K}_{0}$ with the same index, and the bonding map with subscript $i$ in $\mathbf{K}_{1}$ may differ from that in $\mathbf{K}_{0}$ with subscript $i$.

If $i_{0} \in \mathbb{N}, m_{1}<\cdots<m_{i_{0}}$ is a finite sequence in $\mathbb{N}$, and successively we have chosen $\left(\mathbf{K}_{j},\left(\delta_{i}^{j}\right)\right)$ an $m_{j}$-shift of $\left(\mathbf{K}_{j-1},\left(\delta_{i}^{j-1}\right)\right.$ ) from $\Sigma_{j}$ (an appropriate subdivision of the $m_{j}$-term of $\left.\mathbf{K}_{j-1}\right), 1 \leq j \leq i_{0}$, then we may conclude that $\mathbf{K}_{i_{0}}$ is a representation of $X$ which is stable and simplicially irreducible from index $m_{i_{0}}$ with $\left(\delta_{i}^{i_{0}}\right)$ an associated sequence of stability; it satisfies (c) of Definition 3.8 with $\left(\delta_{i}^{\prime}\right)=\left(\delta_{i}^{i_{0}}\right)$ and $\left(\delta_{i}\right)=\left(\delta_{i}^{0}\right)$. The terms (as metric spaces) in $\mathbf{K}_{0}$ and $\mathbf{K}_{i_{0}}$ are equal. For $i<m_{i_{0}}, \delta_{i}^{i_{0}-1}=\delta_{i}^{i_{0}}$, the terms with index $i$ have the same triangulations in $\mathbf{K}_{i_{0}-1}$ and $\mathbf{K}_{i_{0}}$, and the bonding maps in $\mathbf{K}_{i_{0}-1}$ and $\mathbf{K}_{i_{0}}$ with subscript $i$ are equal. For $i \geq m_{i_{0}}$, $\delta_{i}^{i_{0}}$ need not equal $\delta_{i}^{i_{0}-1}$, the triangulation of the term in $\mathbf{K}_{i_{0}}$ with index $i$ is a subdivision of that in $\mathbf{K}_{i_{0}-1}$ with the same index, and the bonding map with subscript $i$ in $\mathbf{K}_{i_{0}}$ may differ from that in $\mathbf{K}_{i_{0}-1}$ with subscript $i$.

In consideration of $(\mathrm{d})$ of Definition 3.8, we may also conclude that if we are given a sequence $\left(\epsilon_{i}\right)$ of positive numbers, then for each $i \in \mathbb{N}$,

$$
d_{i}\left(p_{i}^{i+1}, q_{i}^{i+1}\right)<\epsilon_{i}
$$

where $q_{i}^{i+1}$ denotes the appropriate bonding map from $\mathbf{K}_{i_{0}}, p_{i}^{i+1}$ the one from $\mathbf{K}_{0}$.

Henceforth we typically shall write $\left(\left|K_{(j) i}\right|, p_{(j) i}^{i+1}\right)$ to denote such a representation $\mathbf{K}_{j}, 0 \leq j \leq i_{0}$. One should note that, whenever $i_{0} \geq j_{0} \geq j \geq 1$, then $K_{(j) m_{j}}=$ $K_{\left(j_{0}\right) m_{j}}=\Sigma_{j}$ when this occurs from the procedure in Lemma 3.9.

3.10. Definition. Let $X$ be a metrizable compactum and let $r: \mathbb{N} \rightarrow \mathbb{N}$ be an increasing function. Let $\mathbf{K}_{0}$ be a representation of $X$ which is stable and simplicially irreducible from index $r(1)$ with $\left(\delta_{i}^{0}\right)$ a sequence of stability. Suppose that $\left(\mathbf{K}_{j},\left(\delta_{i}^{j}\right)\right), j \in \mathbb{N}$, is a sequence such that for each $j,\left(\mathbf{K}_{j},\left(\delta_{i}^{j}\right)\right)$ is an $r(j)$-shift of $\left(\mathbf{K}_{j-1},\left(\delta_{i}^{j-1}\right)\right)$ from $\Sigma_{j}$. 
Then for each $k \in \mathbb{N}$, if $m, l$, and $i$ are chosen so that $m \geq l \geq r(k)>i$, one sees that $p_{(l) i}^{i+1}=p_{(m) i}^{i+1}$ and $\delta_{i}^{l}=\delta_{i}^{m}$. So for each $i$, the sequences $\left(\delta_{i}^{j}\right)$ and $\left(p_{(j) i}^{i+1}\right)$ are eventually constant. Hence, in an obvious way, we may define an inverse sequence $\mathbf{K}_{\infty}=\left(\left|K_{(\infty) i}\right|, p_{(\infty) i}^{i+1}\right)=\lim _{j \rightarrow \infty} \mathbf{K}_{j}$, and a sequence $\left(\delta_{i}^{\infty}\right)=\lim _{j \rightarrow \infty}\left(\delta_{i}^{j}\right)$ of positive numbers. Here, $K_{(\infty) i}=\lim _{j \rightarrow \infty} K_{(j) i}$ and $p_{(\infty) i}^{i+1}=\lim _{j \rightarrow \infty} p_{(j) i}^{i+1}$.

The following is not difficult to deduce from our construction and this definition.

3.11. Lemma. Assume the notation of Definition 3.10. Then $\mathbf{K}_{\infty}$ is a representation of $X$. If $i \in \mathbb{N}, g:\left|K_{(\infty) i+1}\right| \rightarrow\left|K_{(\infty) i}\right|$ is a map, and $d_{i}\left(g, p_{(\infty) i}^{i+1}\right)<\delta_{i}^{\infty}$, then $d_{i}\left(g, p_{(0) i}^{i+1}\right)<\delta_{i}^{0}$ and hence $\left(\delta_{i}^{\infty}\right)$ is a sequence of stability for $\mathbf{K}_{\infty}$.

Next is a well-known result which follows from the fact that if $H$ is a countable abelian group, then we may (and always do) express $K(H, n)$ as a countable CWcomplex.

3.12. Lemma. Let $X$ be a compact metrizable space, $H$ a countable abelian group, and $n \in \mathbb{N}$. Then there exists a countable set $\mathcal{H}(X, H)$ of maps of closed subspaces of $X$ to $K(H, n)$ such that for each closed subspace $B$ of $X$ and map $h: B \rightarrow K(H, n)$, there is $h_{0}: B_{0} \rightarrow K(H, n)$ in $\mathcal{H}(X, H)$, where $B \subset B_{0}$ and $h_{0} \mid B \simeq h$.

Recall (see the Introduction) that for each abelian group $H$ and metrizable compactum $X, \operatorname{extdim} X \leq K(H, n)$ is equivalent to the statement $\operatorname{extdim}_{H} X \leq n$. Applying Theorem 1.4 of [Ru2, we may state,

3.13. Lemma. Let $H$ be a countable abelian group, $n \in \mathbb{N}, \mathbf{V}=\left(V_{i}, g_{i}^{i+1}\right)$ an inverse sequence of metrizable compacta, and $V=\lim \mathbf{V}$. Suppose that for each $i, \mathcal{H}\left(V_{i}, H\right)$ as in Lemma 3.12 has been chosen and for each $h: A \rightarrow K(H, n)$ in $\mathcal{H}\left(V_{i}, H\right)$, there exists $k \geq i$ and a map $g: V_{k} \rightarrow K(H, n)$ such that for each $x \in$ $\left(g_{i}^{k}\right)^{-1}(A), h\left(g_{i}^{k}(x)\right)=g(x)$. Then $\operatorname{extdim} V \leq K(H, n)$ and hence $\operatorname{dim}_{H} V \leq n$.

In $\mathrm{Wa}$ the author developed a technique, using an inverse sequence of polyhedra whose limit is a given metrizable compactum $X$, of finding a metrizable compactum $Z$ and a map $\pi: Z \rightarrow X$. Along with this, he showed how to recognize the fibers of $\pi$ as inverse limits of sub-inverse sequences of an inverse sequence whose limit is $Z$. This concept has been refined, and will be quite useful to us in its evolved form. We explain this idea, due to A. Dranishnikov (Dr4] $)$, as follows.

Let $\left(\tilde{K}_{i}, d_{i}\right)$ be a sequence of compact metric spaces having the property that for each $i, \operatorname{diam}_{i} \tilde{K}_{i}<2^{-i}$. Under these conditions, we shall always use the brick metric $\rho$ on $\prod_{i=1}^{\infty} \tilde{K}_{i}$ given by

$$
\rho\left(\left(x_{i}\right),\left(y_{i}\right)\right)=\sum_{i=1}^{\infty} d_{i}\left(x_{i}, y_{i}\right)
$$

In section 3 of Dr4] the author uses this along with the notion of a realization of an inverse sequence $\mathbf{X}=\left(\tilde{K}_{i}, \tilde{p}_{i}^{i+1}\right)$ to obtain an important lemma. Now we state Lemma 4 of [Dr4].

3.14. Lemma. Let $\mathbf{X}=\left(\tilde{K}_{i}, \tilde{p}_{i}^{i+1}\right)$ and $\mathbf{Z}=\left(M_{i}, g_{i}^{i+1}\right)$ be inverse sequences of nonempty metric compacta, $X=\lim \mathbf{X}$ and $Z=\lim \mathbf{Z}$. Assume that for each $i \in \mathbb{N}$, a closed interior cover $\tilde{\mathcal{M}}_{i}$ of $\tilde{K}_{i}$ is given with the additional property that 
no element of $\tilde{\mathcal{M}}_{i}$ equals $\tilde{K}_{i}$ and that for each $x \in \tilde{K}_{i}$, we have selected an element $M_{x, i} \in \tilde{\mathcal{M}}_{i}$ having the property that $B\left(x, \lambda^{u}\left(\tilde{\mathcal{M}}_{i}\right)\right) \subset M_{x, i}$. Suppose also that a map $\phi_{i}: M_{i} \rightarrow \tilde{K}_{i}$ has been chosen such that:

(a) $\phi_{i}^{-1}(M) \neq \emptyset$ for each $M \in \tilde{\mathcal{M}}_{i}$,

(b) $d\left(\tilde{p}_{i}^{i+1} \circ \phi_{i+1}, \phi_{i} \circ g_{i}^{i+1}\right)<\lambda^{u}\left(\tilde{\mathcal{M}}_{i}\right) / 4$, and

(c) $\operatorname{mesh} \tilde{\mathcal{M}}_{i}<\lambda^{u}\left(\tilde{\mathcal{M}}_{i-1}\right) / 4$ when $i>1$.

Then for each $x=\left(x_{i}\right) \in X, g_{i}^{i+1}\left(\phi_{i+1}^{-1}\left(M_{x_{i+1}, i+1}\right)\right) \subset \phi_{i}^{-1}\left(M_{x_{i}, i}\right)$, and there is a surjective map $\pi: Z \rightarrow X$ such that $\pi^{-1}(x)=\lim \mathbf{Z}_{x}$, where

$$
\mathbf{Z}_{x}=\left(\phi_{i}^{-1}\left(M_{x_{i}, i}\right), g_{i}^{i+1}\right) .
$$

\section{Some Lemmas From ALGebra}

The following lemma was motivated by the proof of Fact 2 of the proof of Theorem 3.1 of [KY2].

4.1. Lemma. Let $G$ be an abelian group, let $k \in \mathbb{N}$, and let $u \in G$ be divisible by $q \in \mathbb{N}_{\geq 2}$. Suppose that $r \geq 1$ and $\Delta: \mathbb{Z} \rightarrow \mathbb{Z} \oplus\left(\bigoplus_{1}^{r} G\right)$ is the homomorphism induced by $\Delta(1)=(-q, u,-u,-u, \ldots,-u)$. Then

$$
\left[\mathbb{Z} \oplus\left(\bigoplus_{1}^{r} G\right)\right] / \operatorname{im} \Delta \approx \mathbb{Z} / q \oplus\left(\bigoplus_{1}^{r} G\right) .
$$

Proof. Choose a $z \in G$ such that $q z=u$. Define $h: \mathbb{Z} \oplus\left(\bigoplus_{1}^{r} G\right) \rightarrow \mathbb{Z} / q \oplus\left(\bigoplus_{1}^{r} G\right)$ by

$$
h\left(i, x_{1}, \ldots, x_{r}\right)=\left([i]_{q}, x_{1}+i z, x_{2}+x_{1}, \ldots, x_{r}+x_{1}\right) .
$$

We see that $h$ is a homomorphism because

$$
\begin{aligned}
h\left(i, x_{1}, \ldots, x_{r}\right)+h\left(j, y_{1}, \ldots, y_{r}\right) \\
\quad=\left([i]_{q}, x_{1}+i z, x_{2}+x_{1}, \ldots, x_{r}+x_{1}\right)+\left([j]_{q}, y_{1}+j z, y_{2}+y_{1}, \ldots, y_{r}+y_{1}\right) \\
\quad=\left([i+j]_{q}, x_{1}+y_{1}+(i+j) z, x_{2}+y_{2}+\left(x_{1}+y_{1}\right), \ldots, x_{r}+y_{r}+\left(x_{1}+y_{1}\right)\right) \\
\quad=h\left(\left(i+j, x_{1}+y_{1}, \ldots, x_{r}+y_{r}\right) .\right.
\end{aligned}
$$

Next, we show that $h$ is a surjection. Let $\left(a_{0}, a_{1}, \ldots, a_{r}\right) \in \mathbb{Z} / q \oplus\left(\bigoplus_{1}^{r} G\right)$. Thus $a_{0}=[i]_{q}$ for some $i$. Put $x_{1}=a_{1}-i z$, and $x_{k}=a_{k}-x_{1}$ for $2 \leq k \leq r$. Then $h\left(i, x_{1}, \ldots, x_{r}\right)=\left([i]_{q}, a_{1}-i z+i z, a_{2}, \ldots, a_{r}\right)=\left(a_{0}, a_{1}, \ldots, a_{r}\right)$ as needed.

Now we will detect that $h$ induces an isomorphism as desired by this lemma. To see this, first note that im $\Delta \subset \operatorname{ker} h$ because $h(\Delta(1))=h(-q, u,-u, \ldots,-u)=$ $\left([-q]_{q}, u-q z, u-u, \ldots, u-u\right)=(0, u-u, 0, \ldots, 0)=(0,0, \ldots, 0)$. On the other hand, $\operatorname{ker} h \subset \operatorname{im} \Delta$ for the following reason. Suppose $h\left(i, x_{1}, x_{2}, \ldots, x_{r}\right)=$ $\left([i]_{q}, x_{1}+i z, x_{2}+x_{1}, \ldots, x_{r}+x_{1}\right)=0$. Then $i=q s$ for some $s$. Also, $x_{1}+i z=$ $x_{1}+q s z=x_{1}+s u=0$, showing that $x_{1}=-s u$. For $2 \leq k \leq r, 0=x_{k}+x_{1}=x_{k}-s u$, i.e., $x_{k}=s u$. Finally, $\Delta(-s)=(-q(-s),-s u, s u, \ldots, s u)=\left(i, x_{1}, x_{2}, \ldots, x_{r}\right)$.

Next are some facts from the theory of abelian groups.

4.2. Lemma. Let $p \in \mathbb{P}, q \in \mathbb{N}_{\geq 2}$ and $u \in \mathbb{Z} / p^{\infty}$. Then $u$ is divisible by $q$. 
4.3. Corollary. Let $p \in \mathbb{P}, q \in \mathbb{N}_{\geq 2}, r \in \mathbb{N}, G=\bigoplus_{1}^{r} \mathbb{Z} / p^{\infty}$, and $u \in G$. Then $u$ is divisible by $q$.

Let $P_{0}$ be a subset of $\mathbb{P}$. Recall that the group $\mathbb{Z}_{\left(P_{0}\right)}$ is the set of all elements $b$ of $\mathbb{Q}$ such that when $b=\frac{r}{s}$ reduced to lowest terms, then $s$ has no factors from $P_{0}$.

4.4. Lemma. Let $P_{0} \subset \mathbb{P}$, let $u \in \mathbb{Z}_{\left(P_{0}\right)}$, and let $q$ be a composite of elements of $\mathbb{P} \backslash P_{0}$. Then $u$ is divisible by $q$.

From KY2, we have the following fact.

4.5. Lemma. Let $P_{0} \subset \mathbb{P}$. Then $G=\mathbb{Z}_{\left(P_{0}\right)}$ satisfies the conditions $\left(E W_{1}\right)$ and $\left(E W_{2}\right)$ of $[\mathrm{KY} 2$. In particular, there exists a homomorphism $\alpha: \mathbb{Z} \rightarrow G$ such that

$$
\alpha^{*}: \operatorname{Hom}(G, G) \rightarrow \operatorname{Hom}(\mathbb{Z}, G)
$$

is a surjection.

In order to prove Theorem 1.1 we shall need to form a resolution of the given compactum $X$ in terms of a certain countable set of groups. To detect which groups are needed, we shall apply some Bockstein theory. A good source for this is $\S 2$ of Dr6]; it can also be found in $[\mathrm{Ku}$, but with different notation. For an abelian group $G$, we shall be involved with its Bockstein basis $\sigma(G)$ in an indirect manner. Henceforward we shall denote by $P_{G}$ the set of all $p \in \mathbb{P}$ such that $\mathbb{Z}_{(p)} \in \sigma(G)$.

4.6. Lemma. Let $G$ be an abelian group, $n \in \mathbb{N}$, and $J=\mathbb{Z}_{\left(P_{G}\right)}$. If $X$ is a compact metrizable space and $\operatorname{dim}_{G} X \leq n$, then $\operatorname{dim}_{J} X \leq n, \operatorname{dim}_{\mathbb{Z}_{(p)}} X \leq \operatorname{dim}_{J} X$ for all $p \in P_{G}$, and for some $p^{*} \in P_{G}, \operatorname{dim}_{\mathbb{Z}_{\left(p^{*}\right)}} X=\operatorname{dim}_{J} X$.

Proof. Let $p \in P_{G}$. Since $\mathbb{Z}_{(p)} \in \sigma(G)$, then $\operatorname{dim}_{\mathbb{Z}_{(p)}} X \leq \operatorname{dim}_{G} X \leq n$. On the other hand, $\sigma(J)=\left\{\mathbb{Z}_{(p)} \mid p \in P_{G}\right\}$. Hence $\operatorname{dim}_{\mathbb{Z}_{(p)}} X \leq \operatorname{dim}_{J} X$ for all $p \in P_{G}$, and $\operatorname{dim}_{J} X=\sup \left\{\operatorname{dim}_{\mathbb{Z}_{(p)}} X \mid p \in P_{G}\right\} \leq n$. Thus for some $p^{*} \in P_{G}, \operatorname{dim}_{J} X=$ $\operatorname{dim}_{\mathbb{Z}_{\left(p^{*}\right)}} X \leq n$.

4.7. Definition. Let $G$ be an abelian group. We define a set $\sigma_{0}(G)$ of abelian groups $H$ by requiring that $H \in \sigma_{0}(G)$ if:

(a) $H=\mathbb{Z}_{\left(P_{G}\right)}$,

(b) $H=\mathbb{Z} / p^{\infty}, \mathbb{Z} / p^{\infty} \in \sigma(G), \mathbb{Z} / p \notin \sigma(G)$, and $p \notin P_{G}$, or

(c) $H=\mathbb{Z} / p, \mathbb{Z} / p \in \sigma(G)$, and $p \notin P_{G}$.

4.8. Lemma. Let $G$ be an abelian group, let $H \in \sigma_{0}(G)$ be as in (a) or (b) of Definition 4.7 , let $q \in \mathbb{N}$ be a composite of primes not in $P_{G}$, and let $u \in H$. Then $u$ is divisible by $q$.

Proof. This follows from Lemmas 4.4 and 4.2 .

4.9. Lemma. Let $X$ be a metrizable compactum, let $G$ be an abelian group, let $\sigma_{0}(G)$ be as in Definition 4.7, and let $n \in \mathbb{N}$. Then $\operatorname{dim}_{G} X \leq n$ if and only if $\operatorname{dim}_{H} X \leq n$ for all $H \in \sigma_{0}(G)$.

Proof. Suppose $\operatorname{dim}_{G} X \leq n$. Let $H \in \sigma_{0}(G)$. If $H \in \sigma(G)$, then of course the Bockstein basis theorem shows that $\operatorname{dim}_{H} X \leq n$. If $H \notin \sigma(G)$, then $H$ is of the form $\mathbb{Z}_{\left(P_{G}\right)}$ (see (a) of Definition 4.7). Apply Lemma 4.6 to conclude that $\operatorname{dim}_{H} X \leq n$. 
For the opposite implication, suppose $\operatorname{dim}_{H} X \leq n$ for all $H \in \sigma_{0}(G)$. Let $H^{\prime} \in \sigma(G) \backslash \sigma_{0}(G)$. We simply have to show that $\operatorname{dim}_{H^{\prime}} X \leq n$ (for all such $H^{\prime}$ ).

If for some $p \in \mathbb{P}, H^{\prime}=\mathbb{Z}_{(p)}$, then (a) of Definition 4.7 and Lemma 4.6 prevail. Suppose $H^{\prime}=\mathbb{Q}$. If $P_{G}=\emptyset$, then $\mathbb{Q} \in \sigma_{0}(G)$ by Definition $4.7($ a); hence it must be true that $P_{G} \neq \emptyset$. Pick $p \in P_{G}$. Then Lemma 4.6 shows that $\operatorname{dim}_{\mathbb{Z}_{(p)}} X \leq$ $\operatorname{dim}_{\mathbb{Z}_{\left(P_{G}\right)}} X \leq n$. The Bockstein inequality BI4 of [Dr6] yields $\operatorname{dim}_{\mathbb{Q}} X \leq n$.

Now consider the case $H^{\prime}=\mathbb{Z} / p^{\infty}$ and $p \notin P_{G}$. Since $\mathbb{Z} / p^{\infty} \notin \sigma_{0}(G)$, parts (b) and (c) of Definition 4.7 show that $\mathbb{Z} / p \in \sigma_{0}(G)$. Hence $\operatorname{dim}_{\mathbb{Z} / p} X \leq n$ by assumption. But the Bockstein inequality BI1 in Dr6] tells us that $\operatorname{dim}_{H^{\prime}} X \leq$ $\operatorname{dim}_{\mathbb{Z} / p} X \leq n$. If $p \in P_{G}$, then the Bockstein inequalities BI1 and BI3 along with Lemma 4.6 indicate that $\operatorname{dim}_{H^{\prime}} X \leq \operatorname{dim}_{\mathbb{Z}_{(p)}} X \leq \operatorname{dim}_{\mathbb{Z}_{\left(P_{G}\right)}} X \leq n$.

If for some $p \in \mathbb{P}, H^{\prime}=\mathbb{Z} / p$, then because of (c) of Definition 4.7 , it must be true that $p \in P_{G}$. Apply Bockstein inequality BI3 and Lemma 4.6 again.

For a given abelian group $G$ and metrizable continuum $B$, we are later going to face the problem of establishing, in terms of the groups in $\sigma_{0}(G)$, whether $B$ is $G$-acyclic. The following lemma provides a usable criterion for this.

4.10. Lemma. Given a metrizable continuum $B$, abelian group $G$, and $n \in \mathbb{N}_{\geq 2}$ such that:

(a) $\operatorname{dim}_{G} B \leq n$,

(b) $\check{H}^{k}(B ; H)=0$ for $1 \leq k<n$ and any abelian group $H$,

(c) $\check{H}^{n}(B ; H)=0$ for all $H \in \sigma_{0}(G) \backslash\left\{\mathbb{Z}_{\left(P_{G}\right)}\right\}$, and

(d) $\check{H}^{n}(B ; H)=0$ for any torsion free abelian group $H$.

Then $B$ is $G$-acyclic.

Proof. We are going to show that $B$ is $H$-acyclic for all $H \in \sigma(G)$. The result will thus be true by Corollary 4.7 of $\mathrm{KY} 2$. By (a) and the Bockstein basis theorem, $\operatorname{dim}_{H} B \leq n$. An application of Theorem 1 of $\mathrm{Ku}$ shows that

$$
\check{H}^{n+k}(B ; H)=0 \text { for all } k \geq 1 .
$$

By this and (b) of the hypothesis, we only need to prove that $\check{H}^{n}(B ; H)=0$.

Suppose that $p \in \mathbb{P}$ and $H \in\left\{\mathbb{Z} / p, \mathbb{Z} / p^{\infty}\right\}$. By $(\mathrm{d}), \check{H}^{n}\left(B ; \mathbb{Z}_{(p)}\right)$ is trivial since $\mathbb{Z}_{(p)}$ is torsion free. By $(\mathrm{b}), \check{H}^{k}\left(B ; \mathbb{Z}_{(p)}\right)$ is trivial for $1 \leq k<n$. If $p \in P_{G}$, then $\mathbb{Z}_{(p)} \in \sigma(G)$, so we already know that $\check{H}^{n+k}\left(B ; \mathbb{Z}_{(p)}\right)=0$ for all $k \geq 1$.

Consider the short exact sequence,

$$
0 \rightarrow \mathbb{Z}_{(p)} \stackrel{\times p}{\rightarrow} \mathbb{Z}_{(p)} \rightarrow \mathbb{Z} / p \rightarrow 0 .
$$

(See $[\mathrm{Ku}]$, near the bottom of page 10.) This leads to a long exact sequence in the Cech cohomology of $B$ showing that $\check{H}^{q}(B ; \mathbb{Z} / p)=0$ for all $q>0$. Thus, $B$ is $\mathbb{Z} / p$-acyclic. By Lemma 4.2 of [KY2], $B$ is also $\mathbb{Z} / p^{\infty}$-acyclic. Hence $B$ is $H$-acyclic in this situation.

On the other hand, suppose that $p \notin P_{G}$ and consider first the case that $H=$ $\mathbb{Z} / p$. Then by Definition $4.7(\mathrm{c}), \mathbb{Z} / p \in \sigma_{0}(G)$, so $\check{H}^{n}(B ; H)=0$ because of (c) of the hypothesis. This of course shows that $B$ is $\mathbb{Z} / p$-acyclic. If $H=\mathbb{Z} / p^{\infty}$ and $\mathbb{Z} / p \in \sigma(G)$, then once more Lemma 4.2 of $[\mathrm{KY} 2$ shows that $B$ is $H$-acyclic. So we need to concern ourselves with the case that $\mathbb{Z} / p \notin \sigma(G)$ and $H=\mathbb{Z} / p^{\infty} \in \sigma(G)$. But then Definition 4.7 (b) shows that $\mathbb{Z} / p^{\infty} \in \sigma_{0}(G)$, so again (c) applies and we see that $\check{H}^{n}(B ; H)=0$. 
The last case to consider is that in which $H \in \sigma(G)$ is torsion free; this is covered by (d) of the hypothesis.

4.11. Lemma. Suppose that $G$ is an abelian group, $K$ is a connected $C W$-complex, $n \in \mathbb{N}, \pi_{k}(K)=0$ for $0 \leq k<n$, and $\pi_{n}(K)=G$. Let $Z$ be a metrizable compactum such that $\operatorname{dim} Z \leq n+1$ and $\operatorname{dim}_{G} Z \leq n$. Then $\operatorname{extdim} Z \leq K$.

Proof. We may treat $K=K(G, n)^{(n+1)} \subset K(G, n)$. If $A \subset Z$ is closed and $f: A \rightarrow$ $K$ is a map, then $\operatorname{dim}_{G} Z \leq n$ implies that $f$ extends to a map $F: Z \rightarrow K(G, n)$. The map $F$ may be replaced by a map $F^{*}$ that factors through a polyhedron $|L|$ of dimension $\leq n+1$ in such a manner that $F^{*}(A) \subset K$ and $F$ and $F^{*}$ are homotopic when restricted to maps of $A$ into $K$. Now use a cellular approximation to adjust the map $F^{*}$ so that $F^{*}(Z)$ lands in the $(n+1)$-skeleton of $K(G, n)$, i.e., in $K$. Finally apply the homotopy extension theorem to see that $f$ extends to a map of $Z$ into $K$.

The next lemma may be proved in a manner similar to that of Lemma 4.11.

4.12. Lemma. Suppose that $G$ is an abelian group, $K$ is a connected $C W$-complex, $n \in \mathbb{N}, \pi_{k}(K)=0$ for $0 \leq k<n$ and for $k=n+1$, and $\pi_{n}(K)=G$. Let $Y$ be a metrizable compactum such that $\operatorname{dim} Y \leq n+1$ and $Y$ is $G$-acyclic. Then $Y$ is K-acyclic.

\section{EXtensions OF COMPLEXES}

For the remainder of the paper we shall treat all CW-complexes as being PL and our maps between two of them will be PL. If $e: A \rightarrow B$ is a map of CW-complexes, then by $\operatorname{MCY}(e)$ we mean the mapping cylinder of $e$, where $A$ is identified as a natural subspace in the usual way. When we say "the" generator of a group $G$, we simply mean that the group can be generated by one element and that we have selected a favored generator in advance. Whenever a CW-complex is connected we are going to suppress the notation of a base-point when designating its homotopy groups.

Let us recall that a Moore space $P$ of type $(\mathbb{Z} / q, n), n \in \mathbb{N}, q \in \mathbb{N}_{\geq 2}$, can be described as the quotient space of $B^{n+1}$ under a quotient map which is an embedding of int $B^{n+1}$ and which maps $\partial B^{n+1}$ to $S^{n}$ as a map of degree $q$. Such Moore spaces are treated as CW-complexes and we identify $S^{n}$ as a subspace, denoting it $S_{P}^{n}$. It is well known that

$$
\pi_{k}(P) \approx \begin{cases}0 & \text { if } k<n, \\ \mathbb{Z} / q & \text { if } k=n\end{cases}
$$

and that the inclusion $S_{P}^{n} \hookrightarrow P$ induces the quotient homomorphism $\mathbb{Z} \rightarrow \mathbb{Z} / q$ on $\pi_{n}$. There is a triangulation $L$ of $P$ so that for some at least three times iterated barycentric subdivision $U$ of $B^{n+1}$ (which we treat as an $(n+1)$-simplex), the quotient map $f$ of $B^{n+1}$ to $P$ is simplicial from $U$ to $L$, and so that the $L$-simplicial neighborhood of $S_{P}^{n}$ in $P$ is a regular neighborhood of $S_{P}^{n}$ in $P$. We shall refer to such $L$ as a standard triangulation of $P$.

5.1. Notation. Let $n \in \mathbb{N}$ and $K$ be a simplicial complex. Then $K^{[n+1]}$ will denote $K^{(n+1)} \backslash K^{(n)}$. 
5.2. Definition. Let $n \in \mathbb{N}, K$ be a simplicial complex and $S=K^{(n+1)}$. For each $\sigma \in S^{[n+1]}$, let $e_{\sigma}: \partial \sigma \rightarrow \Theta_{\sigma}$ be an embedding into a connected CW-complex $\Theta_{\sigma}$. Form a CW-complex $M$ by gluing each $\Theta_{\sigma}$ to $\left|S^{(n)}\right|$ using the map, $e_{\sigma}$. We require, of course, that if $\sigma_{1} \neq \sigma_{2}$, then $\Theta_{\sigma_{1}} \cap \Theta_{\sigma_{2}}=\sigma_{1} \cap \sigma_{2}$.

Choose a map $\phi: M \rightarrow|S|$ so that

(a) $\phi=$ id on $\left|S^{(n)}\right|$, and

(b) for each $\sigma \in S^{[n+1]}, \phi^{-1}(\sigma)=\Theta_{\sigma}$.

Let us call the resulting pair $(M, \phi)$ an extension of $K^{(n)}$ associated with $\left\{e_{\sigma} \mid \sigma \in K^{[n+1]}\right\}$. Notice that if desired, we may add the condition

(c) $\phi^{-1}\left(\left|S^{(n)}\right|\right)=\left|S^{(n)}\right|$.

The next lemma was influenced by work in KY2].

5.3. Lemma. Let $n \in \mathbb{N}_{\geq 2}$, let $q \in \mathbb{N}_{\geq 2}$, let $P$ be a Moore space of type $(\mathbb{Z} / q, n)$, let $G$ be an abelian group, and let $u \in G$ be an element which is divisible by $q$. Assume that $L$ is a standard triangulation of $P, \Psi$ is a connected $C W$-complex such that

$$
\pi_{k}(\Psi) \approx \begin{cases}0 & \text { if } k<n, \\ G & \text { if } k=n,\end{cases}
$$

there is a copy $S_{\Psi}^{n}$ of $S^{n}$ lying in $\Psi$, and we have selected an embedding $e_{\tau}$ : $\partial \tau \rightarrow S_{\Psi_{\tau}}^{n}=S_{\Psi}^{n} \subset \Psi_{\tau}=\Psi$ for each $\tau \in L^{[n+1]}$ such that the induced map $H_{n}\left(e_{\tau}\right): H_{n}(\partial \tau ; \mathbb{Z}) \rightarrow H_{n}\left(\Psi_{\tau}, \mathbb{Z}\right)$ carries the generator of $H_{n}(\partial \tau ; \mathbb{Z})$ to $u$ (here we identify $H_{n}(\Psi, \mathbb{Z})$ with $\left.\pi_{n}(\Psi)\right)$. Let $(M, \phi)$ be an extension of $L^{(n)}$ associated with $\left\{e_{\tau} \mid \tau \in L^{[n+1]}\right\}$. Then

$$
\pi_{k}(M) \approx \begin{cases}0 & \text { if } k<n, \\ \mathbb{Z} / q \oplus\left(\bigoplus_{1}^{r} G\right) & \text { if } k=n,\end{cases}
$$

where $r$ is the cardinality of $L^{[n+1]}$.

Proof. It is not difficult to see that $M$ is simply connected. Using a Mayer-Vietoris sequence argument, one can then check that $H_{k}(M ; \mathbb{Z})$ is trivial for all $k<n$. (Begin with $S_{P}^{n}$. Then add the $\Psi_{\tau}$ 's back one by one, so that each new one intersects the union of the preceding sets in a subpolyhedron with homology group $H_{k}=0$.)

This along with the Hurewicz isomorphism theorem show that it is sufficient to demonstrate that $H_{n}(M ; \mathbb{Z}) \approx \mathbb{Z} / q \oplus\left(\bigoplus_{1}^{r} G\right)$. Let $C$ be the simplicial neighborhood of $S_{P}^{n}$ in $P$ under $L$ and let $D$ be the simplicial neighborhood of $\partial B^{n+1}$ in $B^{n+1}$ under $U$ (notation from the beginning of this section). From the above, each is a regular neighborhood.

Choose $\delta_{0} \in U^{[n+1]}$ so that $\partial \delta_{0} \cap D=\emptyset$. Then the closure $D^{\prime}$ of the region between $\delta_{0}$ and $D$ is an annulus which collapses (simplicially) to its intersection with $D$. One sees that $f\left(D^{\prime} \cup D\right)$ collapses to $C$. In turn, $C$ collapses to $S_{P}^{n}$. If we put $\delta_{0}^{\prime}=f\left(\delta_{0}\right)$, then we see by this that the complement of the interior of $\delta_{0}^{\prime}$ in $P$ collapses to $S_{P}^{n}$.

To help understand an argument to follow, suppose that $\delta_{0}, \ldots, \delta_{r-1}$ is a list of the elements of $U^{[n+1]}$. One can see that the $n$-simplexes of $U$ can be oriented in such a manner that the $n$-cycles $\partial \delta_{i}$ and $\partial B^{n+1}$ satisfy the property that $\partial \delta_{0}$ is homologous $($ rel $\mathbb{Z})$ to $\partial \delta_{1}+\cdots+\partial \delta_{r-1}+\partial B^{n+1}$. Thus $H_{n}\left(\left|U^{(n)}\right| ; \mathbb{Z}\right)$ is the 
sum of $r$ copies of $\mathbb{Z}$ and the inclusion of $\partial \delta_{0}$ induces a homomorphism on $n$-th homology which sends the generator of $\partial \delta_{0}$ to the $r$-tuple each of whose entries is the generator of $\mathbb{Z}$.

Now the quotient map $f$ is nondegenerate on elements of $U^{[n+1]}$ since it is a homeomorphism on $\operatorname{int} B^{n+1}$. So it is clear that $f\left(\left|U^{(n)}\right|\right)=\left|L^{(n)}\right|$ and that $f$ carries $\partial B^{n+1}$ onto $S_{P}^{n} \subset P$ by a map of degree $q$. If we label $f\left(\delta_{i}\right)$ as $\delta_{i}^{\prime}$, then we can see by applying the preceding reasoning that $H_{n}\left(\left|L^{(n)}\right| ; \mathbb{Z}\right)$ is isomorphic to the sum of $r$ copies of $\mathbb{Z}$ and that the inclusion $j$ of $\partial \delta_{0}^{\prime}$ induces a homomorphism on $n$-th homology which sends the generator of $H_{n}\left(\partial \delta_{0}^{\prime} ; \mathbb{Z}\right)$ to the $r$-tuple where say the first coordinate is $q$ and each other is just 1 .

Using this information along with an inductive argument involving MayerVietoris sequences, where one considers reversing the collapse of $P \backslash$ int $\delta_{0}^{\prime}$ to $S_{P}^{n}$, we conclude that $M$ may be written as the union of two closed subspaces, say $W_{1}$ and $W_{2}$, such that

(1) $H_{n}\left(W_{1} ; \mathbb{Z}\right) \approx \mathbb{Z} \oplus\left(\oplus_{1}^{r-1} G\right)$,

(2) $H_{n}\left(W_{2} ; \mathbb{Z}\right) \approx G$, and

(3) $W_{1} \cap W_{2}=\partial \delta_{0}^{\prime}$.

Consider the part

$$
H_{n}\left(\partial \delta_{0}^{\prime}\right) \stackrel{\Delta}{\rightarrow} H_{n}\left(W_{1} ; \mathbb{Z}\right) \oplus H_{n}\left(W_{2} ; \mathbb{Z}\right) \rightarrow H_{n}(M) \rightarrow 0
$$

of the Mayer-Vietoris sequence of the pair $\left(W_{1}, W_{2}\right)$. This becomes

$$
\mathbb{Z} \stackrel{\Delta}{\rightarrow} \mathbb{Z} \oplus\left(\bigoplus_{1}^{r} G\right) \rightarrow H_{n}(M) \rightarrow 0
$$

where $\Delta$ is induced by the inclusions $i: \partial \delta_{0}^{\prime} \rightarrow W_{2}, j: \partial \delta_{0}^{\prime} \rightarrow W_{1}$. Clearly $i_{*}(1)=u$. The discussion preceding this leads us to conclude that $j_{*}(1)=(q, u, \ldots, u) \in$ $\mathbb{Z} \oplus\left(\bigoplus_{1}^{r} G\right)$. In the Mayer-Vietoris sequence one employs $-j_{*}$. So our $\Delta$ is the same as the one in Lemma 4.1.

\section{LAYERED EXTENSIONS AND REDUCTIONS}

In this section we are going to describe a method of construction of an extension built as a finite sequence of extensions by the method of section 4 . Beginning with a finite simplicial complex $C_{0}$, there will first be a finite sequence of extensions starting with $C_{0}^{(n)}$, each extension being of the same type involving Moore spaces. Then there will be one more extension which might be construed as a trivial EdwardsWalsh resolution. After doing this we shall look at the construction in reverse, determining recursively some of the homotopy groups of special subcomplexes of it, attaching cells to kill off specific summands of these groups, determining homotopy groups again of certain subcomplexes, attaching cells again, and so forth until we construct an object with desirable characteristics.

For the remainder of this section, assume that $n \in \mathbb{N}_{\geq 2}$ is fixed.

Let $C_{0}$ be a finite simplicial complex with $\operatorname{dim} C_{0} \leq n+1$. Write $M_{0}=\left|C_{0}\right|$. Given $q_{1} \in \mathbb{N}_{\geq 2}$, fix a Moore space $\Theta_{1}$ of type $\left(\mathbb{Z} / q_{1}, n\right)$ along with a collection $\left\{e_{\sigma}: \partial \sigma \rightarrow S_{\Theta_{1}}^{n} \subset \Theta_{1} \mid \sigma \in C_{0}^{[n+1]}\right\}$ of embeddings. Form the extension $\left(M_{1}, \varphi_{1}\right)$ of $C_{0}^{(n)}$ associated with $\left\{e_{\sigma} \mid \sigma \in C_{0}^{[n+1]}\right\}$. 
Suppose we are given $q_{2} \in \mathbb{N}_{\geq 2}$ and a triangulation $C_{1}$ of $M_{1}$ in such a manner that $C_{1} \mid \varphi_{1}^{-1}(\sigma)$ is standard for each $\sigma \in C_{0}^{[n+1]}$. Also require that if $\sigma, \sigma_{1} \in C_{0}^{[n+1]}$, then there is a simplicial isomorphism between $C_{1} \mid \varphi_{1}^{-1}(\sigma)$ and $C_{1} \mid \varphi_{1}^{-1}\left(\sigma_{1}\right)$. Let $\Theta_{2}$ be a Moore space of type $\left(\mathbb{Z} / q_{2}, n\right)$ along with a collection $\left\{e_{\sigma}: \partial \sigma \rightarrow S_{\Theta_{2}}^{n} \subset\right.$ $\left.\Theta_{2} \mid \sigma \in C_{1}^{[n+1]}\right\}$ of embeddings. Form the extension $\left(M_{2}, \varphi_{2}\right)$ of $C_{1}^{(n)}$ associated with $\left\{e_{\sigma} \mid \sigma \in C_{1}^{[n+1]}\right\}$.

Such a procedure may be applied recursively, say $m$ times, finally resulting in an extension $\left(M_{m}, \varphi_{m}\right)$ of $C_{m-1}^{(n)}$ associated with $\left\{e_{\sigma} \mid \sigma \in C_{m-1}^{[n+1]}\right\}$ along with a triangulation $C_{m}$ of $M_{m}$ such that $C_{m} \mid \varphi_{m}^{-1}(\sigma)$ is standard for each $\sigma \in C_{m-1}^{[n+1]}$.

6.1. Definition. We shall refer to such a sequence

$$
M\left(C_{0}\right)=\left(\left(M_{1}, \varphi_{1}\right), \ldots,\left(M_{m}, \varphi_{m}\right)\right)
$$

as an $m$-layer $\left\{q_{1}, \ldots, q_{m}\right\}$-extension of $C_{0}^{(n)}$. Call $\left\{C_{1}, \ldots, C_{m}\right\}$ its triangulations.

6.2. Lemma. Suppose $M\left(C_{0}\right)$ is an m-layer $\left\{q_{1}, \ldots, q_{m}\right\}$-extension of $C_{0}^{(n)}$ with notation as in Definition 6.1. Then for each $1 \leq j \leq m$,

(a) $\operatorname{dim} M_{j} \leq n+1$,

(b) $M_{j-1}^{(n)} \subset M_{j}^{(n)}$,

(c) $M_{j}^{(n)}=\left|C_{j-1}^{(n)}\right|$,

(d) if $\sigma \in C_{j-1}^{[n+1]}$, then $\partial \sigma \subset M_{j}^{(n)}$, and

(e) $M_{j}$ is compact and is connected if $C_{0}$ is connected.

Suppose, moreover, that $V_{0}$ is a subcomplex of $C_{0}$. Put $N_{0}=\left|V_{0}\right|$ and for each $1 \leq i \leq m, N_{i}=\varphi_{i}^{-1}\left(N_{i-1}\right), V_{i}=C_{i} \mid N_{i}$, and $\psi_{i}=\varphi_{i} \mid N_{i}: N_{i} \rightarrow N_{i-1}$. Then,

(f) $\left(\left(N_{1}, \psi_{1}\right), \ldots,\left(N_{m}, \psi_{m}\right)\right)$ is an m-layer $\left\{q_{1}, \ldots, q_{m}\right\}$-extension of $V_{0}$ with triangulations $\left\{V_{1}, \ldots, V_{m}\right\}$.

6.3. Lemma. Let $C_{0}$ be a simplicial complex consisting of an $(n+1)$-simplex $\Delta$ along with all its faces, and let $M\left(C_{0}\right)$ be an m-layer $\left\{q_{1}, \ldots, q_{m}\right\}$-extension of $C_{0}^{(n)}$ with notation as in Definition 6.1. For each $\sigma \in \bigcup_{j=1}^{m} C_{j-1}^{[n+1]}$, let $j_{\sigma}: \partial \sigma \rightarrow$ $M_{m}^{(n)}$ denote the inclusion map and $\alpha_{\sigma} \in \pi_{n}\left(M_{m}^{(n)}\right)$ the image of the generator of $\pi_{n}(\partial \sigma)$ under the induced homomorphism $\pi_{n}\left(j_{\sigma}\right)$. Then, $\left\{\alpha_{\sigma} \mid \sigma \in \bigcup_{j=1}^{m} C_{j-1}^{[n+1]}\right\}$ is a generating set for $\pi_{n}\left(M_{m}^{(n)}\right), \pi_{n}\left(M_{m}\right)$ is a torsion group having nontrivial $q_{m}$ torsion, and $\pi_{k}\left(M_{m}^{(n)}\right)=0$ for $0 \leq k<n$.

Proof. Suppose $m=1$. From Lemma 6.2(c), the fact that $\Delta$ is an $(n+1)$-simplex, the attached object is a Moore space, and since $n \geq 2$, one sees that the lemma is true.

Consider the case $m=2$. Lemma $6.2(\mathrm{e})$ shows that $\pi_{0}\left(M_{m}^{(n)}\right)=0$. We leave to the reader the easily proved fact that $\pi_{1}\left(M_{m}^{(n)}\right)=0$. For the rest of this proof all homology will be with respect to $\mathbb{Z}$. For $\sigma \in \bigcup_{j=1}^{m} C_{j-1}^{[n+1]}$, let $\hat{\alpha}_{\sigma} \in H_{n}\left(M_{m}^{(n)}\right)$ designate the image of the generator of $H_{n}(\partial \sigma)$ in $H_{n}\left(M_{m}^{(n)}\right)$ under the induced homomorphism $H_{n}\left(j_{\sigma}\right)$. We shall show that $H_{k}\left(M_{m}^{(n)}\right)=0$ for $2 \leq k<n$ and 
$\left\{\hat{\alpha}_{\sigma} \mid \sigma \in \bigcup_{j=1}^{m} C_{j-1}^{[n+1]}\right\}$ generates $H_{n}\left(M_{m}^{(n)}\right)$. Then an application of the Hurewicz Theorem $([\mathrm{Hu}])$ would complete the proof about the generating set for $\pi_{n}\left(M_{m}^{(n)}\right)$ for this case.

Clearly $M_{1}$ consists of $M_{1}^{(n)}$ along with some Moore spaces, say $E_{1}, \ldots, E_{r}$ glued respectively along $\partial \sigma_{1}, \ldots, \partial \sigma_{r}$, where $\sigma_{1}, \ldots, \sigma_{r}$ varies in $C_{0}^{[n+1]}$. Each $E_{s}$ has a triangulation $F_{s}$ induced by $C_{1}$. Since $E_{s}$ is a Moore space, then $H_{k}\left(\left|F_{s}^{(n)}\right|\right)=0$, $2 \leq k<n$. Let $\tilde{j}_{s}: \partial \sigma_{s} \rightarrow\left|F_{s}^{(n)}\right|, 1 \leq s \leq r$, and $\tilde{j}_{\tau}: \partial \tau \rightarrow\left|F_{s}^{(n)}\right|, \tau \in F_{s}^{[n+1]}$, denote the inclusion maps. Put $\tilde{\alpha}_{s}$ equal to the image in $H_{n}\left(\left|F_{s}^{(n)}\right|\right)$ of the generator of $H_{n}\left(\partial \alpha_{s}\right)$ under $H_{n}\left(\tilde{j}_{s}\right)$ and for each $\tau \in F_{s}^{[n+1]}$, put $\tilde{\alpha}_{\tau}$ equal to the image in $H_{n}\left(\left|F_{s}^{(n)}\right|\right)$ of the generator of $H_{n}(\partial \tau)$ under $H_{n}\left(\tilde{j}_{\tau}\right)$. Then $\left\{\tilde{\alpha}_{s}\right\} \cup\left\{\tilde{\alpha}_{\tau} \mid \tau \in F_{s}^{[n+1]}\right\}$ is a generating set for $H_{n}\left(\left|F_{s}^{(n)}\right|\right)$.

Now use induction, beginning with $M_{1}^{(n)}$ and successively gluing in $\left|F_{s}^{(n)}\right|, 1 \leq$ $s \leq r$. At each stage use a Mayer-Vietoris sequence to deduce that $H_{k}=0$ for $2 \leq k<n$, and also to detect a generating set for $H_{n}$. At the last instance, one will have found the desired generating set for $\pi_{n}\left(M_{2}^{(n)}\right)$ and that $\pi_{k}\left(M_{2}^{(n)}\right)=0$ for $0 \leq k<n$.

The torsion part follows from the fact that in $H_{n}\left(M_{m}^{(n)}\right), \hat{\alpha}_{\Delta}$ is a multiple of the sum of the other generators, $\hat{\alpha}_{\sigma}$. Of course each of the latter is transformed to an element of torsion in $\pi_{n}\left(M_{m}\right)$ since, geometrically, it includes as the generator of $\pi_{n}$ of an embedded Moore space.

The preceding can be applied recursively; therefore the lemma is true for arbitrary $m \in \mathbb{N}$.

For the rest of this section, assume we have been given a $C_{0}$ and $M\left(C_{0}\right)$ as in Definition 6.1. Also, let $G$ be an abelian group, $u \in G$, and suppose that $u$ is divisible by $q_{i}, 1 \leq i \leq m$. We are going to design, in association with $M\left(C_{0}\right)$ and its specific structure, a sequence $\left(D_{0}, \omega_{0}\right), \ldots,\left(D_{m}, \omega_{m}\right)$ of CW-complexes and maps. This will be arranged so as to decrease the complexity of the homotopy groups of certain subcomplexes of these $D_{k}$ 's, but in a very systematic manner. We shall apply this technology in section 7 below.

Fix a CW-complex $\Phi$ such that $\Phi^{(n-1)}$ is a vertex and

$$
\pi_{k}(\Phi) \approx \begin{cases}0 & \text { if } k<n \\ G & \text { if } k=n\end{cases}
$$

For each $\sigma \in C_{m}^{[n+1]}$, let $d_{\sigma}: \partial \sigma \rightarrow \Phi$ be a map such that $\pi_{n}\left(d_{\sigma}\right)$ sends the generator of $\pi_{n}(\partial \sigma)$ to $u$. Denote by $\Phi_{\sigma}$ the mapping cylinder, $\operatorname{MCY}\left(d_{\sigma}\right)$, and by $e_{\sigma}$ the inclusion of $\partial \sigma$ into $\Phi_{\sigma}$. Select an extension $\left(D_{0}, \omega_{0}\right)$ of $C_{m}^{(n)}$ associated with $\left\{e_{\sigma} \mid \sigma \in C_{m}^{[n+1]}\right\}$.

Recall that $M_{m}$ is endowed with the triangulation $C_{m}$. We may choose a triangulation $T$ of $D_{0}$ and adjust the map $\omega_{0}$ so that

$\left(\mathrm{a}_{1}\right)$ for each $\sigma \in C_{m}^{[n+1]}, T$ triangulates $\omega_{0}^{-1}(\sigma)$, and

$\left(\mathrm{b}_{1}\right) \omega_{0}:|T| \rightarrow\left|C_{m}\right|$ is simplicial. 
Consider now any $\sigma \in C_{m-1}^{[n+1]}$. An application of Lemma 5.3 shows that

$$
\pi_{k}\left(\omega_{0}^{-1}\left(\varphi_{m}^{-1}(\sigma)\right)\right) \approx \begin{cases}0 & \text { if } k<n, \\ \mathbb{Z} / q_{m} \oplus\left(\bigoplus_{1}^{r_{1}} G\right) & \text { if } k=n,\end{cases}
$$

for some $r_{1} \in \mathbb{N}$, where $r_{1}$ is independent of $\sigma$.

Attach an $(n+1)$-cell to $\omega_{0}^{-1}\left(\varphi_{m}^{-1}(\sigma)\right) \subset D_{0}$ via a map on $\partial B^{n+1}$ which induces a homomorphism sending the generator of $\pi_{n}\left(\partial B^{n+1}\right)$ to the generator of the summand $\mathbb{Z} / q_{m}$ of $\pi_{n}\left(\omega_{0}^{-1}\left(\varphi_{m}^{-1}(\sigma)\right)\right)$. Let us designate such an $(n+1)$-cell by $B_{\sigma}$. We shall use $D_{1}$ to denote the CW-complex obtained from $D_{0}$ after attaching all the $(n+1)$-cells $B_{\sigma}$ for $\sigma \in C_{m-1}^{[n+1]}$.

There is a map $\omega_{1}: D_{1} \rightarrow M_{m-1}=\left|C_{m-1}\right|$ which equals $\varphi_{m} \circ \omega_{0}$ on $D_{0}$ and which sends $B_{\sigma}$ into $\sigma$ for each $\sigma \in C_{m-1}^{[n+1]}$. The first thing to note is that

$$
\pi_{k}\left(\omega_{1}^{-1}(\sigma)\right) \approx \begin{cases}0 & \text { if } k<n, \\ \bigoplus_{1}^{r_{1}} G & \text { if } k=n,\end{cases}
$$

for each $\sigma \in C_{m-1}^{[n+1]}$. Indeed, the inclusion induced homomorphism of $\pi_{n}(\partial \sigma)$ into $\pi_{n}\left(\omega_{1}^{-1}(\sigma)\right)$ sends the generator of the former to an element $u^{*}$ of $\bigoplus_{1}^{r_{1}} G$ which is independent of $\sigma$ and so that $u^{*}$ is divisible by $q_{i}, 1 \leq i \leq m$.

The reader should observe that for such $\sigma, \omega_{1}^{-1}(\sigma)=\omega_{0}^{-1}\left(\varphi_{m}^{-1}(\sigma)\right) \cup B_{\sigma}$. Moreover, suppose that $f: B^{n+1} \rightarrow B_{\sigma}$ is a map having the property that $f\left(\partial B^{n+1}\right) \subset$ $\omega_{0}^{-1}\left(\varphi_{m}^{-1}(\sigma)\right)$. Let $\alpha$ be the generator of $\pi_{n}\left(\partial B^{n+1}\right)$ and $g=f \mid \partial B^{n+1}: \partial B^{n+1} \rightarrow$ $\omega_{0}^{-1}\left(\varphi_{m}^{-1}(\sigma)\right)$.

In the quotient map $\mathbb{Z} \rightarrow \mathbb{Z} / q_{m}$, note that $q_{m}=q_{m} \cdot 1$ is mapped to 0 . This shows that

$(\dagger) \pi_{n}(g)(\alpha)$ is divisible by $q_{m}$ in $\pi_{n}\left(\omega_{0}^{-1}\left(\varphi_{m}^{-1}(\sigma)\right)\right)$.

If $g$ is a map of $\partial B^{n+1}$ to $\omega_{0}^{-1}\left(\varphi_{m}^{-1}(\sigma)\right)$ and if $\pi_{n}(g)$ sends the generator of $\pi_{n}\left(\partial B^{n+1}\right)$ to $q_{m} \cdot r$ times the generator of $\mathbb{Z} / q_{m}$, then it sends that generator to zero, so $g$ is nullhomotopic. Hence

(††) if $g$ factors through a map of $\partial B^{n+1}$ to $S^{n}$ of degree which is a multiple of $q_{m}$, then $f \mid \partial B^{n+1}$ extends to a map $f_{0}: B^{n+1} \rightarrow \omega_{0}^{-1}\left(\varphi_{m}^{-1}(\sigma)\right)$.

Now consider $\sigma \in C_{m-2}^{[n+1]}$. This time we apply Lemma 5.3 with the group $G^{\prime}=$ $\bigoplus_{1}^{r_{1}} G$ and the element $u^{*} \in G$ which is divisible by $q_{m-1}$. One concludes that

$$
\pi_{k}\left(\omega_{1}^{-1}\left(\varphi_{m-1}^{-1}(\sigma)\right)\right) \approx \begin{cases}0 & \text { if } k<n, \\ \mathbb{Z} / q_{m-1} \oplus\left(\bigoplus_{1}^{r_{1} r_{2}} G\right) & \text { if } k=n,\end{cases}
$$

for some $r_{2} \in \mathbb{N}$ which is independent of $\sigma$.

Then of course we will form a CW-complex $D_{2}$ by "killing off" the $\mathbb{Z} / q_{m-1}$ summands as in the previous construction by attaching cells denoted $B_{\sigma}, \sigma \in C_{m-2}^{[n+1]}$ respectively to $\omega_{1}^{-1}\left(\varphi_{m-1}^{-1}(\sigma)\right)$, and end with a map $\omega_{2}: D_{2} \rightarrow M_{m-2}=\left|C_{m-2}\right|$ 
similar to $\omega_{1}$ above. One should observe by analogy that

$$
\pi_{k}\left(\omega_{2}^{-1}(\sigma)\right) \approx \begin{cases}0 & \text { if } k<n, \\ r_{1} r_{2} & \text { if } k=n .\end{cases}
$$

Also, $\omega_{2}^{-1}(\sigma)=\omega_{1}^{-1}\left(\varphi_{m-1}^{-1}(\sigma)\right) \cup B_{\sigma}$, and statements similar to $(\dagger)$ and $(\dagger \dagger)$ will be true.

This process obviously can be continued recursively until we have a sequence of maps, $\omega_{j}: D_{j} \rightarrow\left|C_{m-j}\right|, 0 \leq j \leq m$. In particular several conditions will be true of this construction. Suppose that $1 \leq j \leq m$; then

(1) $\left|C_{0}^{(n)}\right| \subset D_{m}, D_{j-1} \subset D_{j}$ and $D_{j} \backslash D_{j-1}=\bigcup\left\{\operatorname{int} B_{\sigma} \mid \sigma \in C_{m-j}^{[n+1]}\right\}$,

(2) $\omega_{j} \mid D_{j-1}=\varphi_{m-j+1} \circ \omega_{j-1}$

(3) for each $\sigma \in C_{m-j}^{[n+1]}, \omega_{j}^{-1}(\sigma)=\omega_{j-1}^{-1}\left(\varphi_{m-j+1}^{-1}(\sigma)\right) \cup B_{\sigma}$, and

(4) for each $\sigma \in C_{0}^{[n+1]}$, there exists $r \in \mathbb{N}$ (which may depend on $\sigma$ ) such that

$$
\pi_{k}\left(\omega_{m}^{-1}(\sigma)\right) \approx \begin{cases}0 & \text { if } k<n, \\ \bigoplus_{1}^{r} G & \text { if } k=n .\end{cases}
$$

Moreover, suppose that $\sigma \in C_{m-j}^{[n+1]}$ and $f: B^{n+1} \rightarrow B_{\sigma}$ is a map having the property that $f\left(\partial B^{n+1}\right) \subset \omega_{j-1}^{-1}\left(\varphi_{m-j+1}^{-1}(\sigma)\right)$. Then

(5) $f \mid \partial B^{n+1}: \partial B^{n+1} \rightarrow \omega_{j-1}^{-1}\left(\varphi_{m-j+1}^{-1}(\sigma)\right)$ is of degree a multiple of $q_{m-j+1}$, and

(6) if $f \mid \partial B^{n+1}$ factors through a map of $\partial B^{n+1}$ to $S^{n}$ of degree which is a multiple of $q_{m-j+1}$, then $f \mid \partial B^{n+1}$ extends to a map $f_{0}: B^{n+1} \rightarrow$ $\omega_{j-1}^{-1}\left(\varphi_{m-j+1}^{-1}(\sigma)\right)$.

6.4. Definition. We shall refer to a sequence $\left(\left(D_{0}, \omega_{0}\right), \ldots,\left(D_{m}, \omega_{m}\right)\right)$ of the type just constructed, satisfying (1)-(6), as a reduction of $M\left(C_{0}\right)$ for (the abelian group) $G$.

6.5. Lemma. Assume that $\left\{q_{1}, \ldots, q_{m}\right\} \subset \mathbb{N}_{\geq 2}, G$ is an abelian group, $u \in$ $G$, and $u$ is divisible by $q_{i}$ for each $i$. Then for each simplicial complex $C_{0}$ with $\operatorname{dim} C_{0} \leq n+1$ and $m$-layer $\left\{q_{1}, \ldots, q_{m}\right\}$-extension $M\left(C_{0}\right)$ with triangulations $C_{1}, \ldots, C_{m}$, there exists a reduction $\left(\left(D_{0}, \omega_{0}\right), \ldots,\left(D_{m}, \omega_{m}\right)\right)$ of $M\left(C_{0}\right)$ for $G$. For each $\sigma \in C_{m}^{[n+1]}, \omega_{0}^{-1}(\sigma)=\operatorname{MCY}\left(d_{\sigma}\right)$, where $d_{\sigma}: \partial \sigma \rightarrow \Phi$, and $\pi_{n}\left(d_{\sigma}\right)$ carries the generator of $\pi_{n}(\partial \sigma)$ to $u \in G \approx \pi_{n}(\Phi)$. The $C W$-complex $\Phi$ is chosen so that

$$
\pi_{k}(\Phi) \approx \begin{cases}0 & \text { if } k<n, \\ G & \text { if } k=n .\end{cases}
$$

6.6. Lemma. Assume that $q \in \mathbb{N}_{\geq 2}$ and $G=\mathbb{Z} / q$. Then for each simplicial complex $C_{0}$ with $\operatorname{dim} C_{0} \leq n+1$ and $m$-layer $\left\{q_{1}, \ldots, q_{m}\right\}$-extension $M\left(C_{0}\right)$, there exists a reduction $\left(\left(D_{0}, \omega_{0}\right), \ldots,\left(D_{m}, \omega_{m}\right)\right)$ of $M\left(C_{0}\right)$ for $G$.

Proof. We only need to make a few adjustments to the construction preceding Definition 6.4. In producing $D_{0}$, this time let $\Phi$ be a Moore space of type of $(\mathbb{Z} / q, n), d_{\sigma}$ sending generator to generator. Then, up to a homotopy equivalence, $\left(\left(M_{1}, \varphi_{1}\right), \ldots,\left(M_{m}, \varphi_{m}\right),\left(D_{0}, \omega_{0}\right)\right)$ is an $(m+1)$-layer $\left\{q_{1}, \ldots, q_{m}, q\right\}$-extension of $C_{0}^{(n)}$. Applying Lemmas 6.2(f) and 6.3, for each $\sigma \in C_{m-1}^{[n+1]}, \pi_{n}\left(\omega_{0}^{-1}\left(\varphi_{m}^{-1}(\sigma)\right)\right)$ is 
a finitely generated torsion group with nontrivial $q$-torsion. This time attach an $(n+1)$-cell $B_{\sigma}$ to kill all but the $q$-torsion. This process can be continued recursively until the desired conclusion is reached.

\section{Main CONSTRUCtion SteP}

Our proof of Theorem 1.1 in section 8 will involve an inductive construction. There is one main, quite technical step in that procedure, which we would like to present in isolation now.

For the remainder of this section, assume that $n \in \mathbb{N}, \mathbf{Y}=\left(\left|L_{i}\right|, p_{i}^{i+1}\right)$ is an inverse sequence of finite polyhedra, $Y=\lim \mathbf{Y}$, and for each $i \in \mathbb{N}$,

(i) $\operatorname{dim} L_{i} \leq n+1$, and

(ii) $p_{i}^{i+1}:\left|\bar{L}_{i+1}\right| \rightarrow\left|L_{i}\right|$ is a simplicial surjection.

Put $C_{0}=L_{1}$, and suppose that $M\left(C_{0}\right)=\left(\left(M_{1}, \varphi_{1}\right), \ldots,\left(M_{m}, \varphi_{m}\right)\right)$ is an $m$-layer $\left\{q_{1}, \ldots, q_{m}\right\}$-extension of $C_{0}^{(n)}$ (see Definition 6.1), with triangulations $C_{i}, 1 \leq i \leq$ $m$. Assume that we are given a subcomplex $N$ of $C_{m}$ with

(iii) $C_{m}^{(n)} \subset N$.

7.1. Lemma. Let $P_{0} \subset \mathbb{P}$ and $\left\{q_{0}, q_{1}, \ldots, q_{m}\right\} \subset \mathbb{N}$ be selected so that each $q_{i}$ is a composite of elements of $\mathbb{P} \backslash P_{0}$, and either $G=\mathbb{Z}_{\left(P_{0}\right)}$ or for some $p \in \mathbb{P} \backslash P_{0}$, $G \in\left\{\mathbb{Z} / p, \mathbb{Z} / p^{\infty}\right\}$. Assume that $\operatorname{dim}_{G} Y \leq n$. Then there exists $s>1$, such that for all $\epsilon>0$ there is a subdivision $V_{0}$ of $L_{s}$, an $(m+1)$-layer $\left\{q_{1}^{\prime}, \ldots, q_{m+1}^{\prime}\right\}$ extension $M\left(V_{0}^{(n+1)}\right)=\left(\left(N_{1}, \psi_{1}\right), \ldots,\left(N_{m+1}, \psi_{m+1}\right)\right)$ of $V_{0}^{(n)}$ with triangulations $V_{i}, 1 \leq i \leq m+1$, and a map $g: N_{m+1} \rightarrow M_{m}$ such that:

(a) $\operatorname{mesh} V_{0}<\epsilon$,

(b) $q_{i}^{\prime}$ is a composite of elements of $\mathbb{P} \backslash P_{0}$, and $q_{0}$ divides $q_{i}^{\prime}$ for each $1 \leq i \leq$ $m+1$,

(c) for each $\sigma_{0} \in \bigcup_{j=1}^{m} V_{j-1}^{[n+1]}, g \mid \partial \sigma_{0}: \partial \sigma_{0} \rightarrow M_{m}$ is of degree a multiple of $q_{0}$,

(d) if $G \neq \mathbb{Z}_{\left(P_{0}\right)}$ and $\sigma^{\prime} \in V_{m}$, then either $\psi_{m+1}^{-1}\left(\sigma^{\prime}\right) \subset g^{-1}(|N|)$ or $\sigma^{\prime} \in V_{m}^{[n+1]}$ and $\psi_{m+1}^{-1}\left(\sigma^{\prime}\right) \cap g^{-1}(|N|)=\partial \sigma^{\prime}$, and

(f) in case $G=\mathbb{Z}_{\left(P_{0}\right)}$ and $h:|N| \rightarrow K(G, n)$ is a map, then $h \circ g \mid g^{-1}(|N|)$ : $g^{-1}(|N|) \rightarrow K(G, n)$ extends to a map of $N_{m+1}$ to $K(G, n)$.

Moreover, let $\varphi=\varphi_{1} \circ \cdots \circ \varphi_{m}: M_{m} \rightarrow\left|L_{1}\right|$ and $\psi=\psi_{1} \circ \cdots \circ \psi_{m+1}: N_{m+1} \rightarrow\left|L_{s}\right|$ :

(g) then for each $z \in N_{m+1}$, there is a principal simplex $\sigma^{\#}$ of $L_{1}$ such that if $p_{1}^{s} \circ \psi(z)$ lies in $\sigma^{\#}$, then $\varphi \circ g(z) \in \sigma^{\#}$.

Proof. Let us use $p_{k}: Y \rightarrow\left|L_{k}\right|$ for the coordinate projections. Observe that because of (ii),

(1) for each $k \geq 1$, the coordinate projection $p_{k}$ is surjective.

If $G \in\left\{\mathbb{Z} / p^{\infty}, \mathbb{Z}_{\left(P_{0}\right)}\right\}$, then we shall pick an element $u \in G$ as follows. Should $G=\mathbb{Z} / p^{\infty}$, choose $u=0 \in G$. Otherwise select $\alpha: \mathbb{Z} \rightarrow \mathbb{Z}_{\left(P_{0}\right)}$ as in Lemma 4.5, and choose $u=\alpha(1)$. In either case (see Lemma 4.4), the hypotheses of Lemma 6.5 apply. If $G=\mathbb{Z} / p$, then Lemma 6.6 comes into play. Hence we may select a reduction $\left(\left(D_{0}, \omega_{0}\right),\left(D_{1}, \omega_{1}\right), \ldots,\left(D_{m}, \omega_{m}\right)\right)$ of $M\left(C_{0}\right)$ for $G$. Let us agree that if $G=\mathbb{Z}_{\left(P_{0}\right)}$, then in forming $\left(D_{0}, \omega_{0}\right)$ we shall use a $C W$-complex $\Phi$ as in Lemma 6.5 , and so that $u=\alpha(1) \in G \approx \pi_{n}(\Phi)$ is as above. 
As a consequence of (i), $\operatorname{dim} Y \leq n+1$, and we are given that $\operatorname{dim}_{G} Y \leq n$. One then may apply (4) in Definition 6.4 along with Lemma 2.5 to see that extdim $Y \leq$ $\omega_{m}^{-1}(\sigma)$ for each $\sigma \in C_{0}^{[n+1]}$. Putting it another way, we may say that

(2) for each $\sigma \in C_{0}^{[n+1]}, \omega_{m}^{-1}(\sigma)$ is an absolute extensor for $Y$.

We claim that there exists $s>1$ and a map $\beta_{0}:\left|L_{s}\right| \rightarrow D_{m}$ such that

(3) if $y \in Y$ and $p_{1}(y) \in \sigma$, where $\sigma$ is a principal simplex of $L_{1}$, then $\beta_{0} \circ p_{s}(y) \in$ $\omega_{m}^{-1}(\sigma)$.

We shall first get a map $\gamma: Y \rightarrow D_{m}$. Applying (1) of Definition 6.4, define $\gamma$ on $p_{1}^{-1}\left(C_{0}^{(n)}\right)$ to equal $p_{1}$. Now if $\sigma \in C_{0}^{[n+1]}$, consider $W_{\sigma}=\omega_{m}^{-1}(\sigma)$. Then $\gamma\left(p_{1}^{-1}(\partial \sigma)\right)=p_{1}\left(p_{1}^{-1}(\partial \sigma)\right) \subset \partial \sigma \subset W_{\sigma}$. Using (2), extend $\gamma$ to a map of $p_{1}^{-1}(\sigma)$ into $W_{\sigma}$. Put all these extensions together to get $\gamma: Y \rightarrow D_{m}$ so that

(4) if $y \in Y$ and $p_{1}(y) \in \sigma$, where $\sigma$ is a principal simplex of $C_{0}$, then $\gamma(y) \in$ $\omega_{m}^{-1}(\sigma)$

There exists $s>1$ and a map $\beta_{0}:\left|L_{s}\right| \rightarrow D_{m}$ such that the composition $\beta_{0} \circ p_{s}$ is as close to $\gamma$ as we wish. For each principal simplex $\sigma$ of $C_{0}, \omega^{-1}(\sigma)$ is a subcomplex of $D_{m}$. So in light of (4) modify the map $\beta_{0}$ if necessary so that (3) is true.

As a consequence of (3) and (1), we may conclude that

(5) if $t \in\left|L_{s}\right|$ and $p_{1}^{s}(t) \in \sigma$, where $\sigma$ is a principal simplex of $L_{1}$, then $\beta_{0}(t) \in$ $\omega_{m}^{-1}(\sigma)$

Next we shall begin the construction of the objects $\left(N_{k}, \psi_{k}\right), 1 \leq k \leq m+1$. The first $m$ steps of this program are designed to "peel off" the cells $B_{\sigma}$ from the target space in a certain gradual manner. We will start by replacing $\beta_{0}:\left|L_{s}\right| \rightarrow D_{m}$ by a map $\beta_{1}: N_{1} \rightarrow D_{m-1}$ and then proceed recursively so that in the end, no $B_{\sigma}$ will lie in the image of the last map $\beta_{m}: N_{m} \rightarrow D_{0}$.

Now we apply (1) of Definition 6.4. Taking into account (5) above, one may choose a subdivision $V_{0}$ of $L_{s}$ satisfying (a), perhaps adjust the map $\beta_{0}$ slightly, and conclude that

(6) $\beta_{0}\left(\left|V_{0}^{(n)}\right|\right) \subset D_{0}$

(7) if $\sigma_{0} \in V_{0}$, then for some principal simplex $\sigma_{0}^{\#} \in C_{0}, \beta_{0}\left(\sigma_{0}\right) \subset \omega_{m}^{-1}\left(\sigma_{0}^{\#}\right)$, and

(8) if $\sigma_{0} \in V_{0}^{[n+1]}$, then either $\beta_{0}\left(\sigma_{0}\right) \subset D_{m-1}$ or $\beta_{0}\left(\sigma_{0}\right)=B_{\sigma}$ for some $\sigma \in C_{0}^{[n+1]}$.

We claim that

(9) for each $\sigma_{0} \in V_{0}^{[n+1]}, \beta_{0} \mid \partial \sigma_{0}: \partial \sigma_{0} \rightarrow D_{m-1}$ is of degree a multiple of $q_{m}$.

According to (8), there are but two possibilities. In case $\beta_{0}\left(\sigma_{0}\right) \subset D_{m-1}$, then (9) is true because $\beta_{0} \mid \partial \sigma_{0}: \partial \sigma_{0} \rightarrow D_{m-1}$ is nullhomotopic. If $\beta_{0}\left(\sigma_{0}\right)=B_{\sigma}$ for some $\sigma \in C_{0}^{[n+1]}$, then an application of (5) of Definition 6.4 along with (6) of this section yields (9).

Choose

$$
q_{1}^{\prime}=q_{0} q_{m}
$$

Put $N_{0}=\left|V_{0}\right|$. Fix a Moore space $\Phi_{1}$ of type $\left(\mathbb{Z} / q_{1}^{\prime}, n\right)$ along with a collection $\left\{e_{\sigma}: \partial \sigma \rightarrow S_{\Phi_{1}}^{n} \subset \Phi_{1} \mid \sigma \in V_{0}^{[n+1]}\right\}$ of embeddings. Form the extension $\left(N_{1}, \psi_{1}\right)$ associated with $\left\{e_{\sigma} \mid \sigma \in V_{0}^{[n+1]}\right\}$. 
A map $\beta_{1}: N_{1} \rightarrow D_{m-1}$, having the property

(10) if $\sigma_{0} \in V_{0}$, then for some principal simplex $\sigma_{0}^{\#} \in C_{0}, \beta_{1}\left(\psi_{1}^{-1}\left(\sigma_{0}\right)\right) \subset$ $\omega_{m-1}^{-1}\left(\varphi_{1}^{-1}\left(\sigma_{0}^{\#}\right)\right)$

is described as follows. Consider $\sigma_{0} \in V_{0}$. We will define $\beta_{1}$ in two mutually exclusive, exhaustive cases. First if $\beta_{0}\left(\sigma_{0}\right) \subset D_{m-1}$, then on $\psi_{1}^{-1}\left(\sigma_{0}\right)$, put $\beta_{1}=$ $\beta_{0} \circ \psi_{1}$. Then (10) holds because of (7) and item (2) of Definition 6.4.

In the other situation, we first define $\beta_{1}=\beta_{0} \circ \psi_{1}=\beta_{0}$ on $\partial \sigma_{0}$. We then use (6) of Definition 6.4 , with $f$ replaced by $\beta_{0}$, to get an extension of $\beta_{1}$ to the entire Moore space $\psi_{1}^{-1}\left(\sigma_{0}\right)$, thought of as a quotient of $B^{n+1}$ under a certain map which has degree $q_{1}^{\prime}$ on $\partial B^{n+1}$. One sees from this definition that again (10) is true. Also, (9) obtains if we replace $\beta_{0}$ by $\beta_{1}$, i.e.,

(11) for each $\sigma_{0} \in V_{0}^{[n+1]}, \beta_{1} \mid \partial \sigma_{0}: \partial \sigma_{0} \rightarrow D_{m-1}$ is of degree a multiple of $q_{m}$. Surely one effect of this construction is to "peel off" the cells $B_{\sigma}, \sigma \in C_{0}^{[n+1]}$, from the image of this new map $\beta_{1}$.

We may select a triangulation $V_{1}$ of $N_{1}$, slightly (up to homotopy) modify the map $\beta_{1}: N_{1} \rightarrow D_{m-1}$ if necessary, and conclude that along with (10) and (11),

(12) $\beta_{1}\left(\left|V_{1}^{(n)}\right|\right) \subset D_{0}$, and

(13) if $\sigma_{0} \in V_{1}^{[n+1]}$, then either $\beta_{1}\left(\sigma_{0}\right) \subset D_{m-2}$ or $\beta_{1}\left(\sigma_{0}\right)=B_{\sigma}$ for some $\sigma \in C_{1}^{[n+1]}$.

Now choose

$$
q_{2}^{\prime}=q_{0} q_{m-1}
$$

Fix a Moore space $\Phi_{2}$ of type $\left(\mathbb{Z} / q_{2}^{\prime}, n\right)$ along with a collection $\left\{e_{\sigma}: \partial \sigma \rightarrow S_{\Phi_{2}}^{n} \subset\right.$ $\left.\Phi_{2} \mid \sigma \in V_{1}^{[n+1]}\right\}$ of embeddings, and form the extension $\left(N_{2}, \psi_{2}\right)$ associated with $\left\{e_{\sigma} \mid \sigma \in V_{1}^{[n+1]}\right\}$. There is no loss of generality in assuming that $V_{1} \mid \psi_{1}^{-1}(\sigma)$ is standard for each $\sigma \in V_{0}^{[n+1]}$.

Replacing $\beta_{0}$ by $\beta_{1}$, apply a procedure just like the one used above to get a map $\beta_{2}: N_{2} \rightarrow D_{m-2}$, agreeing with $\beta_{1}$ on $\left|V_{1}^{(n)}\right|$ and satisfying:

(14) for each $\sigma_{0} \in \bigcup_{j=0}^{1} V_{j}^{[n+1]}, \beta_{2} \mid \partial \sigma_{0}: \partial \sigma_{0} \rightarrow D_{m-2}$ is of degree a multiple of $q_{m-1}$, and

(15) if $\sigma_{0} \in V_{0}$, then for some principal simplex $\sigma_{0}^{\#} \in C_{0}, \beta_{2}\left(\psi_{2}^{-1}\left(\psi_{1}^{-1}\left(\sigma_{0}\right)\right)\right) \subset$ $\omega_{m-2}^{-1}\left(\varphi_{2}^{-1}\left(\varphi_{1}^{-1}\left(\sigma_{0}^{\#}\right)\right)\right)$

Such a process may be applied recursively, resulting in extensions $\left(N_{1}, \psi_{1}\right), \ldots$, $\left(N_{m}, \psi_{m}\right)$, triangulations $V_{1}, \ldots, V_{m-1}$ (we have not yet selected $\left.V_{m}\right)$, maps $\beta_{1}, \ldots$, $\beta_{m}\left(\beta_{i}: N_{i} \rightarrow D_{m-i}\right)$, and $q_{1}^{\prime}, \ldots, q_{m}^{\prime}$. The following conditions will hold true from this process:

(16) $q_{0}$ divides $q_{i}^{\prime}$ for each $1 \leq i \leq m$,

(17) for each $\sigma_{0} \in \bigcup_{j=1}^{m} V_{j-1}^{[n+1]}, \beta_{m} \mid \partial \sigma_{0}: \partial \sigma_{0} \rightarrow D_{0}$ is of degree a multiple of $q_{i}$ for some $1 \leq i \leq m$,

(18) if $\sigma_{0} \in V_{0}$, then for some principal simplex $\sigma_{0}^{\#}$ of $C_{0}$,

$$
\begin{aligned}
& \beta_{m}\left(\left(\psi_{1} \circ \cdots \circ \psi_{m}\right)^{-1}\left(\sigma_{0}\right)\right) \subset \omega_{0}^{-1}\left(\left(\varphi_{1} \circ \cdots \circ \varphi_{m}\right)^{-1}\left(\sigma_{0}^{\#}\right)\right)=\omega_{0}^{-1}\left(\varphi^{-1}\left(\sigma_{0}^{\#}\right)\right), \\
& \text { and } \\
& \text { (19) } V_{i} \mid \psi_{i}^{-1}(\sigma) \text { is standard for each } \sigma \in V_{i-1}^{[n+1]} \text { and } 1 \leq i \leq m .
\end{aligned}
$$


Recall from section $6\left(\right.$ see $\left(\mathrm{a}_{1}\right)$ and $\left.\left(\mathrm{b}_{1}\right)\right)$ that $D_{0}$ may be assigned a triangulation $T$ and $\omega_{0}$ chosen so that $\omega_{0}:|T| \rightarrow\left|C_{m}\right|$ is simplicial. Thus we may impose a triangulation $V_{m}$ on $N_{m}$ so that $V_{m} \mid \psi_{m}^{-1}(\sigma)$ is standard for each $\sigma \in V_{m-1}$, and we may assume (by a simplicial approximation) that, along with (17) and (18), $\beta_{m}:\left|V_{m}\right| \rightarrow|T|$ is simplicial. Hence we may make the above choices of $\omega_{0}$ and $\beta_{m}$ so that

(20) if $G \neq \mathbb{Z}_{\left(P_{0}\right)}$, the map $\omega_{0} \circ \beta_{m}:\left|V_{m}\right| \rightarrow\left|C_{m}\right|$ is simplicial.

By applying (c) of Definition 5.2, we require that

(21) if $G=\mathbb{Z}_{\left(P_{0}\right)}$, then $\omega_{0}^{-1}\left(\left|C_{m}^{(n)}\right|\right)=\left|C_{m}^{(n)}\right|$.

We put a triangulation $V_{m}$ on $N_{m}$ so that $V_{m} \mid \psi_{m}^{-1}(\sigma)$ is standard for each $\sigma \in V_{m-1}$.

Set $q_{m+1}^{\prime}=q_{0}$ and let $\Phi_{m+1}$ be a Moore space of type $\left(\mathbb{Z} / q_{m+1}^{\prime}, n\right)$. Choose a collection $\left\{e_{\sigma}: \partial \sigma \rightarrow S_{\Phi_{m+1}}^{n} \subset \Phi_{m+1} \mid \sigma \in V_{m}^{[n+1]}\right\}$ of embeddings, and form the extension $\left(N_{m+1}, \psi_{m+1}\right)$ associated with $\left\{e_{\sigma} \mid \sigma \in V_{m}^{[n+1]}\right\}$. We require now, for this extension, the condition (c) of Definition 5.2, i.e.,

(22) $\psi_{m+1}^{-1}\left(\left|V_{m}^{(n)}\right|\right)=\left|V_{m}^{(n)}\right|$.

We have already established (a), and certainly our choice of the numbers $q_{1}^{\prime}, \ldots$, $q_{m+1}^{\prime}$ meets the requirement (b) of this lemma. We define

$$
g=\omega_{0} \circ \beta_{m} \circ \psi_{m+1}: N_{m+1} \rightarrow M_{m} .
$$

An application of (17) and the fact that $\beta_{m}$ is a factor in the composition defining $g$ show that (c) is true. One arrives at (d) because of (20), (22), and since $N$ is a subcomplex of $C_{m}$ containing $C_{m}^{(n)}$.

For (f), we examine $h \circ \omega_{0} \mid \omega_{0}^{-1}(|N|): \omega_{0}^{-1}(|N|) \rightarrow K(G, n)$. Now the CWcomplex $\Phi$ may be thought of as a subcomplex of $K(G, n)$ since it was chosen as in Lemma 6.5. If $\sigma \in C_{m}^{[n+1]}$, then (21), our choice of $\alpha$, and an application of Lemma 4.5 show that if $\sigma \notin N$, then the map $h \circ \omega_{0} \mid \partial \sigma$ extends to a map of $\omega_{0}^{-1}(\sigma)=\Phi$ to $K(G, n)$. Since $\omega_{0}$ is a factor of $g$, then (f) follows.

The last statement, (g), can be traced from (18), the definition of $\psi_{m+1}$, and the factorization of $g$.

\section{Proof of the Main theorem}

Proof of Theorem 1.1. Recall from section 4 that $P_{G}=\left\{p \in \mathbb{P} \mid \mathbb{Z}_{(p)} \in \sigma(G)\right\}$. If $P_{G}=\mathbb{P}$, then an application of Example 1 in section 2 (page 8) of [Dr6] shows that $\operatorname{dim}_{\mathbb{Z}} X \leq n$. In that case, we could apply the Edwards-Walsh resolution theorem Wa to get a cell-like resolution $\pi: Z \rightarrow X$, where $\operatorname{dim} Z \leq n$; of course each fiber of $\pi$ would be $G$-acyclic. Hence (a)-(c) of Theorem 1.1 would be satisfied in an even stronger manner. Thus, let us continue under the assumption that $\mathbb{P} \backslash P_{G} \neq \emptyset$.

Choose a function $\mu: \mathbb{N} \rightarrow \mathbb{N} \times \mathbb{N}$ which is surjective and each of whose fibers is infinite, always denoting $\mu(i)$ as $\left(u_{i}, v_{i}\right)$. We do this so that

(1) $u_{i} \leq i$ for each $i$.

We may assume that $\operatorname{card} X \geq 2$. Using Lemma 3.7, let $\mathbf{K}_{0}=\left(\left|K_{(0) i}\right|, p_{(0) i}^{i+1}\right)$ be a stably irreducible representation of $X$ which is simplicial from index 1 . For each $k \in \mathbb{N}$, let

$$
\mathcal{M}_{k}=\overline{\operatorname{st}} K_{(0) k}
$$


We are going to describe a certain recursive process in which there will be an increasing function $r: \mathbb{N} \rightarrow \mathbb{N}, r(1)=1$. This will show us where to make a sequence of $r(i)$-shifts beginning with $\mathbf{K}_{0}$. There will also be an inverse sequence $\left(M_{i}, g_{i}^{i+1}\right)$, where each $M_{i}$ is a finite polyhedron with $\operatorname{dim} M_{i} \leq n+1$. The limit of this inverse sequence will be the space $Z$, but in order to produce the requested map $\pi: Z \rightarrow X$, it will be necessary to construct the sequence carefully, providing a complicated set of inductive hypotheses along the way.

We shall use the convention that whenever we have determined a space $M_{i}$, then $g_{i}^{i}: M_{i} \rightarrow M_{i}$ will denote the identity map. Begin the process by choosing $r(1)=1$. Let $\Sigma_{1}$ be a subdivision of $K_{(0) 1}$ such that mesh $\Sigma_{1}<\lambda^{u}\left(\mathcal{M}_{1}\right) / 4$. Define $S_{1}$ to be the restriction of $\Sigma_{1}$ to $\left|K_{(0), 1}^{(n+1)}\right|$, i.e.,

$$
S_{1}=\Sigma_{1}|| K_{(0), 1}^{(n+1)} \mid
$$

Select $q_{1,1}$ to be the first element of $\mathbb{P} \backslash P_{G}$. For each $\sigma \in S_{1}^{[n+1]}$, let $\Theta_{\sigma}$ be a Moore space of type $\left(\mathbb{Z} / q_{1,1}, n\right)$ and let $e_{\sigma}: \partial \sigma \rightarrow S_{\Theta_{\sigma}}^{n} \subset \Theta_{\sigma}$ be an embedding. Let $\left(M_{1,1}, \phi_{1,1}\right)$ be an extension (Definition 5.2) of $S_{1}^{(n)}$ associated with $\left\{e_{\sigma} \mid \sigma \in\right.$ $\left.S_{1}^{[n+1]}\right\}$. Define

$$
M_{1}=M_{1,1} \text { and } \phi_{1}=\phi_{1,1}: M_{1} \rightarrow\left|S_{1}\right| \text {. }
$$

Select a countable collection $\mathcal{H}_{1}=\bigcup\left\{\mathcal{H}\left(M_{1}, H\right) \mid H \in \sigma_{0}(G)\right\}=\left\{h_{1}^{j}: A_{1}^{j} \rightarrow\right.$ $\left.K\left(H_{1}^{j}, n\right) \mid j \in \mathbb{N}\right\}$ of maps using Lemma 3.12.

Because of (1), it must be true that $u_{1}=1$; consider the map $h_{u_{1}}^{v_{1}}=h_{1}^{v_{1}}: A_{u_{1}}^{v_{1}}=$ $A_{1}^{v_{1}} \rightarrow K\left(H_{u_{1}}^{v_{1}}, n\right)$ from the closed subspace $A_{u_{1}}^{v_{1}}$ of $M_{1}$. Put $C_{1,0}=S_{1}$. There exists a triangulation $C_{1,1}$ of $M_{1}$ such that for each $\sigma \in C_{1,0}^{[n+1]}, \phi_{1,1}^{-1}(\sigma)$ is triangulated by $C_{1,1}$ in a standard manner (see the text preceding Notation 5.1). We arrange this so that for some subcomplex $N_{1}$ of $C_{1,1},\left|C_{1,1}^{(n)}\right| \cup A_{u_{1}}^{v_{1}} \subset\left|N_{1}\right|$, and there is a map $\lambda_{1}:\left|N_{1}\right| \rightarrow K\left(H_{u_{1}}^{v_{1}}, n\right)$ which is an extension of $h_{u_{1}}^{v_{1}}$.

Notice that we have created (Definition 6.1) a 1-layer $\left\{q_{1,1}\right\}$-extension $M\left(C_{1,0}\right)=$ $\left(\left(M_{1,1}, \phi_{1,1}\right)\right)$, of $S_{1}^{(n)}$, whose triangulation is $C_{1,1}$. Apply Definition 3.8 with $\Sigma=$ $\Sigma_{1}$ to get a 1-shift; let $\mathbf{K}_{1}=\left(K_{(1) i}, p_{(1) i}^{i+1}\right)$ denote the resulting inverse sequence.

Now we are ready to make our inductive statement. Suppose $w \in \mathbb{N}$ and we have chosen $1=r(1)<\cdots<r(w)$ in $\mathbb{N}$ and for each $1 \leq k \leq w$, successive $r(k)$-shifts from $\Sigma_{k}$ as in Lemma 3.9. From this we get a finite sequence $\mathbf{K}_{0}, \ldots, \mathbf{K}_{w}$ of inverse sequences with the notation (see the text before Definition 3.10) $\mathbf{K}_{k}=\left(K_{(k) i}, p_{(k) i}^{i+1}\right)$. We always define

$$
S_{k}=\Sigma_{k}|| K_{(0), r(k)}^{(n+1)} \mid .
$$

Let us require that for each $k$ we have determined a finite $\mathrm{CW}$-complex $M_{k}$ with $\operatorname{dim} M_{k} \leq n+1$, and a map $\phi_{k}: M_{k} \rightarrow\left|S_{k}\right|$ so that:

(I1) $\operatorname{mesh} \Sigma_{k}<\lambda^{u}\left(\mathcal{M}_{r(k)}\right) / 4$;

(I2) if $1<k$, there is a map $g_{k-1}^{k}: M_{k} \rightarrow M_{k-1}$ so that

$$
d_{r(k-1)}\left(\phi_{k-1} \circ g_{k-1}^{k}, p_{(k-1) r(k-1)}^{r(k)} \circ \phi_{k}\right)<\lambda^{u}\left(\mathcal{M}_{r(k)}\right) / 4 ;
$$

(I3) we have chosen a collection $\mathcal{H}_{k}=\bigcup\left\{\mathcal{H}\left(M_{k}, H\right) \mid H \in \sigma_{0}(G)\right\}=\left\{h_{k}^{j}: A_{k}^{j} \rightarrow\right.$ $\left.K\left(H_{k}^{j}, n\right) \mid j \in \mathbb{N}\right\}$ of maps as in Lemma 3.12; and

(I4) when $k<w$ we have selected a map $\Lambda_{k+1}: M_{k+1} \rightarrow K\left(H_{u_{k}}^{v_{k}}, n\right)$ such that $\Lambda_{k+1}\left|\left(g_{u_{k}}^{k+1}\right)^{-1}\left(A_{u_{k}}^{v_{k}}\right)=h_{u_{k}}^{v_{k}} \circ g_{u_{k}}^{k+1}\right|\left(g_{u_{k}}^{k+1}\right)^{-1}\left(A_{u_{k}}^{v_{k}}\right)$. 
Putting $C_{k, 0}=S_{k}$, we require further that for some $\left\{q_{k, 1}, \ldots, q_{k, k}\right\} \subset \mathbb{N}$, there is a $k$-layer $\left\{q_{k, 1}, \ldots, q_{k, k}\right\}$-extension $M\left(C_{k, 0}\right)=\left(\left(M_{k, 1}, \phi_{k, 1}\right), \ldots,\left(M_{k, k}, \phi_{k, k}\right)\right)$ of $C_{k, 0}^{(n)}$ with triangulations $\left\{C_{k, 1}, \ldots, C_{k, k}\right\}$ such that

(I5) for some subcomplex $N_{k}$ of $C_{k, k},\left|C_{k, k}^{(n)}\right| \cup\left(g_{u_{k}}^{k}\right)^{-1}\left(A_{u_{k}}^{v_{k}}\right) \subset\left|N_{k}\right|$, and there is a map $\lambda_{k}:\left|N_{k}\right| \rightarrow K\left(H_{u_{k}}^{v_{k}}, n\right)$ which is an extension of $h_{u_{k}}^{v_{k} \circ g_{u_{k}}^{k}} \mid\left(g_{u_{k}}^{k}\right)^{-1}\left(A_{u_{k}}^{v_{k}}\right)$,

(I6) each $q_{k, i}$ is a composite of elements of $\mathbb{P} \backslash P_{G}$,

(I7) $M_{k}=M_{k, k}$,

(I8) $\phi_{k}=\phi_{k, 1} \circ \cdots \circ \phi_{k, k}: M_{k} \rightarrow\left|S_{k}\right|$, and

(I9) if $1<k$ and $H_{u_{k-1}}^{v_{k-1}} \in\left\{\mathbb{Z} / p, \mathbb{Z} / p^{\infty}\right\}$, then for each $\sigma \in \bigcup_{j=1}^{k-1} C_{k, j-1}^{[n+1]}, g_{k-1}^{k} \mid \partial \sigma$ : $\partial \sigma \rightarrow M_{k-1}$ is a map whose degree is a multiple of $p^{k-1}$.

The reader may check that we have satisfied the inductive requirements in case $w=1$. We define the inverse sequence

$$
\mathbf{Y}_{w}=\left(\left|S_{w}\right| \stackrel{p_{(w) r(w)}^{r(w)+1}}{\longleftarrow}\left|K_{(w) r(w)+1}^{*}\right| \stackrel{p_{(w) r(w)+1}^{r(w)+2}}{\longleftarrow}\left|K_{(w) r(w)+2}^{*}\right| \leftarrow \ldots\right),
$$

where $K_{(w) r(w)+1}^{*}$ is the $(n+1)$-skeleton of $\left(p_{(w) r(w)}^{r(w)+1}\right)^{-1}\left(S_{w}\right), K_{(w) r(w)+2}^{*}$ is the $(n+1)$-skeleton of $\left(p_{(w) r(w)+1}^{r(w)+2}\right)^{-1}\left(K_{(w) r(w)+1}^{*}\right)$, and so forth. The bonding maps should of course be the appropriate restrictions of the ones indicated. Lemma 3.9 and Definition 3.6 show that the bonding maps of $\mathbf{Y}_{w}$ are simplicial surjections. Let $Y=\lim \mathbf{Y}_{w}$. Since $Y$ embeds in $X=\lim \mathbf{K}_{w}$, then $\operatorname{dim}_{G} Y \leq n$. Hence $\mathbf{Y}_{w}$ meets the conditions (i) and (ii) specified in section 7 .

The conditions following (ii) in section 7 and immediately preceding Lemma 7.1 are currently met by the data from the induction as follows:

(2) $C_{0}=C_{w, 0}$,

(3) $M\left(C_{w, 0}\right)=\left(\left(M_{w, 1}, \phi_{w, 1}, \ldots,\left(M_{w, w}, \phi_{w, w}\right\}\right)\right.$, a $w$-layer $\left\{q_{w, 1}, \ldots, q_{w, w}\right\}$ extension of $C_{w, 0}$, and

(4) $N=N_{w}$ ((iii) of section 7).

If $H_{u_{w}}^{v_{w}}=\mathbb{Z} / p$, then choose $q_{w, 0}=p^{w+1}$. If $H_{u_{w}}^{v_{w}}=\mathbb{Z} / p^{\infty}$, then note that for some $w_{0} \in \mathbb{N}, \lambda_{w}\left(\left|N_{w}\right|\right) \subset K\left(\mathbb{Z} / p^{w_{0}}, n\right) \subset K\left(\mathbb{Z} / p^{\infty}, n\right)$. In that case, choose $q_{w, 0}=$ $\max \left\{p^{w+1}, p^{w_{0}}\right\}$. Otherwise, let $q_{w, 0}$ be the first element of $\mathbb{P} \backslash P_{G}$. Now let us apply Lemma 7.1 with $\left\{q_{w, 0}, q_{w, 1}, \ldots, q_{w, w}\right\} \subset \mathbb{N}$ and $G=H_{u_{w}}^{v_{w}}$. We put $r(w+1)=s$, where $s>r(w)$ comes from Lemma 7.1. Using (a) of that lemma, there is a subdivision $\Sigma_{w+1}$ (denoted $V_{0}$ in Lemma 7.1) of $K_{(w) r(w+1)}$ so that (I1) is true for $k=w+1$. Then with $C_{w+1,0}=S_{w+1}$, find a $(w+1)$-layer $\left\{q_{w+1,1}, \ldots, q_{w+1, w+1}\right\}$ extension $M\left(C_{w+1,0}\right)=\left(\left(M_{w+1,1}, \phi_{w+1,1}\right), \ldots,\left(M_{w+1, w+1}, \phi_{w+1, w+1}\right)\right)$ of $C_{w+1,0}^{(n)}$ with triangulations $C_{w+1, i}, 1 \leq i \leq w+1$, along with a map $g: M_{w+1, w+1} \rightarrow M_{w}$ as in Lemma 7.1. Define

$$
M_{w+1}=M_{w+1, w+1}
$$

and put

$$
g_{w}^{w+1}: M_{w+1} \rightarrow M_{w}
$$

equal the map $g$ from Lemma 7.1. Define

$$
\phi_{w+1}=\phi_{w+1,1} \circ \cdots \circ \phi_{w+1, w+1}: M_{w+1} \rightarrow\left|S_{w+1}\right|
$$

precisely as required by (I8). 
In our application of Lemma 7.1, the complex $L_{1}$ is in fact $S_{w}$, which is a subcomplex of $\Sigma_{w}$. Thus (I1) for $k=w$ yields that mesh $S_{w}<\lambda^{u}\left(\mathcal{M}_{r(w)}\right) / 4$, so, (g) of Lemma 7.1 shows that the commutativity desired in (I2) is true for $k=w+1$. Select a collection $\mathcal{H}_{w+1}$ so that (I3) is satisfied. We have to find a map $\Lambda_{w+1}$ to meet the condition in (I4).

In case $H_{u_{w}}^{v_{w}}=\mathbb{Z}_{\left(P_{G}\right)}$, consider in (I5), for $k=w$, the subcomplex $N_{w}$ of $C_{w, w}$ and the map $\lambda_{w}:\left|N_{w}\right| \rightarrow K\left(H_{u_{w}}^{v_{w}}, n\right)$. Simply apply (f) of Lemma 7.1 to get the needed extension $\Lambda_{w+1}$ in (I4).

When $H_{u_{w}}^{v_{w}} \in\left\{\mathbb{Z} / p, \mathbb{Z} / p^{\infty}\right\}$ we need a different approach. This time we shall apply (d) of Lemma 7.1. For $x \in\left(g_{w}^{w+1}\right)^{-1}\left(\left|N_{w}\right|\right)$ we of course define $\Lambda_{w+1}(x)=$ $\lambda_{w} \circ g_{w}^{w+1}(x)$. It remains, according to (d) of Lemma 7.1, to extend $\Lambda_{w+1}$ to sets of the form $\phi_{w+1, w+1}^{-1}\left(\sigma^{\prime}\right)$, where $\sigma^{\prime} \in C_{w+1, w}^{[n+1]}$ and $\phi_{w+1, w+1}^{-1}\left(\sigma^{\prime}\right) \cap\left(g_{w}^{w+1}\right)^{-1}\left(\left|N_{w}\right|\right)=$ $\partial \sigma^{\prime}$. But $\phi_{w+1, w+1}^{-1}\left(\sigma^{\prime}\right)$ is a Moore space of type $\left(\mathbb{Z} / q_{w+1, w+1}, n\right)$, where $q_{w+1, w+1}$ is divisible by $q_{w, 0}$. If $H_{u_{w}}^{v_{w}}=\mathbb{Z} / p$, then from the fact that $q_{w, 0}$ is divisible by $p$, we see that $\Lambda_{w+1}$ extends to a map of $\phi_{w+1, w+1}^{-1}\left(\sigma^{\prime}\right)$ to $K\left(H_{u_{w}}^{v_{w}}, n\right)$. If $H_{u_{w}}^{v_{w}}=\mathbb{Z} / p^{\infty}$, then since $\lambda_{w}\left(\left|N_{w}\right|\right) \subset K\left(\mathbb{Z} / p^{w_{0}}, n\right)$ and $q_{w, 0}$ is divisible by $p^{w_{0}}$, we get the needed extension in this situation also. So (I4) is satisfied.

We have already chosen the extension $M\left(C_{w+1,0}\right)$ and the triangulations $\left\{C_{w+1,1}\right.$, $\left.\ldots, C_{w+1, w+1}\right\}$, but if we take just a bit more care in choosing $C_{w+1, w+1}$, we can be certain of the existence of a subcomplex $N_{w+1}$ and a map $\lambda_{w+1}$ as required by (I5). Both (I6) and (I7) can easily be verified from our construction. What remains is (I9). This, however, follows from (c) of Lemma 7.1 and the fact that under these choices of the group $H_{u_{w}}^{v_{w}}, q_{w, 0}$ is divisible by $p^{w+1}$.

Our inductive construction is complete.

We have selected a sequence $\left(\mathbf{K}_{i}\right)$ satisfying the conditions of Definition 3.10, so let $\mathbf{K}_{\infty}$ denote its limit. Using Lemma 3.11 , one sees that $X=\lim \mathbf{K}_{\infty}$. To prepare for the use of Lemma 3.14, for each $i \in \mathbb{N}$ set

(5) $\tilde{K}_{i}=\left|K_{(0) r(i)}\right|, \tilde{\mathcal{M}}_{i}=\mathcal{M}_{r(i)}, \tilde{p}_{i}^{i+1}=p_{(i) r(i)}^{r(i+1)}$, and $\mathbf{X}=\left(\tilde{K}_{i}, \tilde{p}_{i}^{i+1}\right)$.

We have already constructed $\left(M_{i}, g_{i}^{i+1}\right)$ and $\phi_{i}: M_{i} \rightarrow \tilde{K}_{i}$.

There is no loss of generality in taking $X=\lim \mathbf{X}$. For each $i \in \mathbb{N}$ and $x \in \tilde{K}_{i}$, by the definition of $\lambda^{u}$, there exists $M_{x, i} \in \tilde{\mathcal{M}}_{i}=\overline{\operatorname{st}} K_{(0) r(i)}$ as required in Lemma 3.14. For a given $M=M_{x, i} \in \tilde{\mathcal{M}}_{i}$, conditions (f) and (b) of Lemma 6.2 imply that each vertex in $M$ lies in $\phi_{i}^{-1}(M)$; so (a) of Lemma 3.14 is satisfied. We get (b) from the first part of (I2). By Definition 3.6, $\mathbf{K}_{0}$ satisfies (c) of Lemma 3.4, so an application of Lemma 3.5 gives us the condition (c) of Lemma 3.14. Thus there is a surjective map $\pi: Z=\lim \mathbf{Z} \rightarrow X=\lim \mathbf{X}$ whose fibers $\lim \mathbf{Z}_{x}$ are as indicated in Lemma 3.14.

We claim that the metrizable compactum $Z$ and the surjective map $\pi: Z \rightarrow X$ will satisfy the list (a)-(c) of Theorem 1.1. To begin, since $\operatorname{dim} M_{i} \leq n+1$ for each $i$, it is a standard fact that the limit $Z$ of the sequence of $M_{i}$ 's has $\operatorname{dim} \leq n+1$. So $(b)$ is true.

For (c) we only have to show that $\operatorname{dim}_{G} Z \leq n$ because of (b) of the theorem (the part we just proved) and Lemma 4.11. We shall prove this by using Lemma 4.9. Let $H \in \sigma_{0}(G)$. The idea is to use Lemma 3.13 with the countable abelian group $H$ and the inverse sequence $\mathbf{Z}$ defining the space $Z$. So let $i \in \mathbb{N}$ and consider an element of $\mathcal{H}\left(M_{i}, H\right)$, say $h_{i}^{j}: A_{i}^{j} \rightarrow K\left(H_{i}^{j}, n\right)$ as in (I3). There exists $k_{0} \geq i$ 
so that $\mu\left(k_{0}\right)=(i, j)$; thus $i=u_{k_{0}}$ and $j=v_{k_{0}}$. We just apply (I4) to obtain $k=k_{0}+1$ leading to the extension requested in Lemma 3.13.

We have to prove (a) of Theorem 1.1. Fix $x=\left(x_{i}\right) \in X$; we know from Lemma 3.14 (look for $\mathbf{Z}_{x}$ ) that $\pi^{-1}(x)$ can be written as the limit of an inverse sequence of the form $\left(\phi_{i}^{-1}\left(M_{x_{i}, i}\right), g_{i}^{i+1}\right)$, where $M_{x_{i}, i}=\overline{\operatorname{st}}\left(w_{i}, K_{(0) r(i)}\right) \in \tilde{\mathcal{M}}_{i}$ for some vertex $w_{i}$ of $K_{(0) r(i)}$. But since the image of $\phi_{i}$ is $\left|S_{i}\right|=\left|K_{(0) r(i)}^{(n+1)}\right|$, then we may replace $M_{x_{i}, i}$ by $F_{i}=\overline{\operatorname{st}}\left(w_{i}, K_{(0) r(i)}^{(n+1)}\right)$ in the preceding formulation. This means that $\pi^{-1}(x)=$ $\lim \left(\phi_{i}^{-1}\left(F_{i}\right), g_{i}^{i+1}\right)$.

Thinking of $F_{i}$ as a CW-complex, one sees that $F_{i}^{(n)}$ is homotopy equivalent to the union of the $n$-skeleta of the $(n+1)$-simplexes of $K_{(0) r(i)}$ contained in it. For each such $(n+1)$-simplex $\sigma, S_{i}$ induces a triangulation $S_{\sigma}$ on $\sigma$. Observe also that $F_{i}$ may be treated, up to homotopy equivalence, as a bouquet of $(n+1)$-cells in 1-1 correspondence with the $(n+1)$-simplexes $\sigma$ just mentioned. From our construction, one can ascertain that $\phi_{i}^{-1}\left(F_{i}\right)$ is, up to homotopy equivalence, a bouquet, say $B_{i}$, of the objects $\phi_{i}^{-1}(\sigma)$. According to the last part of Lemma 6.3, each of the objects $\left[\phi_{i}^{-1}(\sigma)\right]^{(n)}$ has trivial homotopy groups in the dimensions $0 \leq k<n$. It follows then that $\pi_{k}\left(B_{i}^{(n)}\right)=0$ for $0 \leq k<n$. From this information we may conclude that for each $i \in \mathbb{N}$ and $0 \leq k<n$,

(6) the inclusion $B_{i}^{(k)} \hookrightarrow B_{i}^{(k+1)}$ is nullhomotopic.

So it is not difficult to show that for $0<k<n$ and any abelian group $H$, every map of $\phi_{i}^{-1}\left(F_{i}\right)$ to $K(H, k)$ is nullhomotopic. Hence one concludes that

(7) $\tilde{\tilde{H}}^{k}\left(\pi^{-1}(x) ; H\right)=0$ for $0 \leq k<n$ and all abelian groups $H$.

For dimension $n$ the strategy is a bit different. We shall show that

(8) if $p \in \mathbb{P}$ and either $Z / p$ or $\mathbb{Z} / p^{\infty}$ lies in $\sigma_{0}(G)$, then for all $j \in \mathbb{N}$, $\check{H}^{n}\left(\pi^{-1}(x) ; \mathbb{Z} / p^{j}\right)=0$.

Then surely $\check{H}^{n}\left(\pi^{-1}(x) ; \mathbb{Z} / p\right)=0$, but also

(9) $\check{H}^{n}\left(\pi^{-1}(x) ; \mathbb{Z} / p^{\infty}\right)=0$

by the following reasoning. Let us recall that for any compact metrizable space $P$ and abelian group $H, \check{H}^{n}(P ; H)=0$ if and only if every map of $P$ to $K(H, n)$ is nullhomotopic. Since $\pi^{-1}(x)$ is compact, every map of it to $K\left(\mathbb{Z} / p^{\infty}, n\right)$ lands in some $K\left(\mathbb{Z} / p^{j}, n\right)$. From (8), we see that such a map must be nullhomotopic as a map into $K\left(\mathbb{Z} / p^{j}, n\right) \subset K\left(\mathbb{Z} / p^{\infty}, n\right)$.

Let us assume that $p \in \mathbb{P}$ and either $\mathbb{Z} / p$ or $\mathbb{Z} / p^{\infty} \in \sigma_{0}(G)$. Fix $j \in \mathbb{N}$ and let $H$ be either $\mathbb{Z} / p^{j}$ or a torsion free group. We are going to show that

(10) for all $k \in \mathbb{N}$, there exists $i \geq k$ such that for any map $\varphi: \phi_{i}^{-1}\left(F_{i}\right) \rightarrow$ $K(H, n)$, the composition $\varphi \circ g_{i}^{i+1}: \phi_{i+1}^{-1}\left(F_{i+1}\right) \rightarrow K(H, n)$ is nullhomotopic.

This will show that the induced homomorphism

$$
H^{n}\left(g_{i}^{i+1}\right): H^{n}\left(\phi_{i}^{-1}\left(F_{i}\right) ; H\right) \rightarrow H^{n}\left(\phi_{i+1}^{-1}\left(F_{i+1}\right) ; H\right)
$$

is trivial. That would surely prove (8), but at the same time it would yield

(11) $\check{H}^{n}\left(\pi^{-1}(x) ; H\right)=0$ for any torsion free abelian group $H$.

Thus we are going to establish (10). 
We claim that

(12) for all $k \in \mathbb{N}$, there exists $i \geq k$ such that for any map $h: S^{n} \rightarrow \phi_{i+1}^{-1}\left(F_{i+1}\right)$, the composition $\varphi \circ g_{i}^{i+1} \circ h: S^{n} \rightarrow K(H, n)$ is nullhomotopic.

Let us see why (12) would imply (10) and then see how to prove (12).

Because of (6), there exists a homotopy $G^{*}: B_{i+1}^{(n-1)} \times I \rightarrow B_{i+1}^{(n)}$, where $G_{0}^{*}$ is the inclusion and $G_{1}^{*}$ is a constant map, say to $p_{0}$. Let $\tau$ be an $n$-cell of $B_{i+1}$. We determine a map $d_{\tau}: \partial(\tau \times I) \rightarrow B_{i+1}^{(n)}$ by setting $d_{\tau}(t, s)=t$ if $s=0, G^{*}(t, s)$ if $t \in$ $\partial \tau$, and $p_{0}$ if $s=1$. Then the map $\varphi \circ g_{i}^{i+1} \circ d_{\tau}: \partial(\tau \times I) \rightarrow K(H, n)$ is nullhomotopic by (12). So the map $\varphi \circ g_{i}^{i+1} \circ d_{\tau}$ extends to a map $D_{\tau}: \tau \times I \rightarrow K\left(\mathbb{Z} / p^{j}, n\right)$. A map $D: B_{i+1}^{(n)} \times I \rightarrow K(H, n)$ is defined by setting $D=\bigcup\left\{D_{\tau} \mid \tau\right.$ an $n$-cell of $\left.B_{i+1}\right\}$. One notes that $D$ is a nullhomotopy of the map $\varphi \circ g_{i}^{i+1} \mid B_{i+1}^{(n)}$. Since $\pi_{n+1}(K(H, n))=0$, this nullhomotopy extends to one of $B_{i+1}^{(n+1)} \times I$ to $K(H, n)$. This proves (10) modulo (12).

Now for (12). In case $H$ is a torsion free group, e.g., $H=\mathbb{Z}_{\left(P_{G}\right)}$ in our case, then choose $i=k$. It follows from (I7) and Lemma 6.3 that $\pi_{n}\left(\phi_{i+1}^{-1}\left(F_{i+1}\right)\right)$ is a torsion group. So we simply have to note that $H$ is a torsion free group.

Suppose that $H=\mathbb{Z} / p^{j}$; then we may assume without loss of generality that $k \geq j$. There exists $l$ such that $H_{k}^{l} \in\left\{\mathbb{Z} / p, \mathbb{Z} / p^{\infty}\right\}$. Hence there is $i \geq k \geq j$ such that $\mu(i)=(k, l)$, i.e., $u_{i}=k$ and $v_{i}=l$. From (I9), one sees that for each $\sigma \in \bigcup_{j_{0}=1}^{i} C_{i+1, j_{0}-1}^{[n+1]}, g_{i}^{i+1}$ carries $\partial \sigma$ to $M_{i}$ by a map whose degree is a multiple of $p^{j}$. So it must be true that $\varphi \circ g_{i}^{i+1}$ carries $\partial \sigma$ to $K\left(\mathbb{Z} / p^{j}, n\right)$ by a map whose degree is a multiple of $p^{j}$. This and an application of Lemma 6.3 show that for any map $h$ as in (12), the composition $\varphi \circ g_{i}^{i+1} \circ h$ has degree which is a multiple of $p^{j}$. Such a map has to be nullhomotopic. Our proof of (12) is complete.

Now we shall establish that each fiber $B$ of the map $\pi$ is $G$-acyclic. We already have proved that $\operatorname{dim}_{G} Z \leq n$, so $\operatorname{dim}_{G} B \leq n$, that is, (a) of Lemma 4.10 is certainly true. One obtains (b), (c), and (d) from (7), (8), (9), and (11).

Since $\pi^{-1}(x) \subset Z$ and $\operatorname{dim}_{G} Z \leq n$, then $\operatorname{dim}_{G}\left(\pi^{-1}(x)\right) \leq n$. Hence an application of Theorem 1 of $\left[\mathrm{Ku}\right.$ ] shows that $\check{H}^{n+k}\left(\pi^{-1}(x) ; G\right)=0$ for all $k \geq 1$. This completes our proof of (a) of Theorem 1.1. In case $\pi_{n+1}(K)=0$, then (aa) is assured by an application of Lemma 4.12 since each fiber of $\pi$ is $G$-acyclic and has $\operatorname{dim} \leq n+1$.

\section{REFERENCES}

[Dr1] A. Dranishnikov, On P. S. Aleksandrov's problem, Mat. Sb. 135 (4) (1988), 551-557. MR0942139 (90e:55004)

[Dr2] A. Dranishnikov, On homological dimension modulo $p$, Math. USSR Sbornik 60, no. 2 (1988), 413-425. MR0889602 (88h:55001)

[Dr3] A. Dranishnikov, Homological dimension theory, Russian Math. Surveys 43(4) (1988), 11-63. MR0969565 (90e:55003)

[Dr4] A. Dranishnikov, K-theory of Eilenberg-Mac Lane spaces and cell-like mapping problem, Trans. Amer. Math. Soc. 335:1 (1993), 91-103. MR.1153012 (93e:55003)

[Dr5] A. Dranishnikov, Rational homology manifolds and rational resolutions, Topology and its Appls. 94 (1999), 75-86. MR1695349 (2000j:55001)

[Dr6] A. Dranishnikov, Cohomological dimension theory of compact metric spaces, preprint.

[DD] A. Dranishnikov and J. Dydak, Extension dimension and extension types, Tr. Mat. Inst. Steklova 212 (1996), 61-94. MR1635023(99h:54049) 
[Dy] J. Dydak, Cohomological dimension theory, Handbook of Geometric Topology, Elsevier, Amsterdam, 2002, pp. 423-470. MR.1886675 (2003a:55002)

[DW] J. Dydak and J. Walsh, Complexes that arise in cohomological dimension theory: a unified approach, J. London Math. Soc. (2) (48), no. 2 (1993), 329-347. MR.1231719|(94d:55003)

[Ed] R. D. Edwards, A theorem and question related to cohomological dimension and cell-like maps, Notices of the Amer. Math. Soc. 25 (1978), A-259.

[Hu] S. Hu, Homotopy Theory, Academic Press, New York, 1959. MR0106454 (21:5186)

[JR] R. Jimenez and L. Rubin, An addition theorem for $n$-fundamental dimension in metric compacta, Topology and its Appls. 62 (1995), 281-297. MR.1326827 (96a:54026)

[KY1] A. Koyama and K. Yokoi, A unified approach of characterizations and resolutions for cohomological dimension modulo p, Tsukuba J. Math. 18(2) (1994), 247-282. MR1305817 (95j:55002)

[KY2] A. Koyama and K. Yokoi, Cohomological dimension and acyclic resolutions, Topology and its Appls. 120 (2002), 175-204. MR1895491 (2003b:55001)

[Ku] W. I. Kuz'minov, Homological Dimension Theory, Russian Math. Surveys 23 (1968), 1-45. MR0240813 (39:2158)

[Le] M. Levin, Acyclic resolutions for arbitrary groups, Isr. J. Math. 135 (2003), 193-204. MR 1997043 (2004f:55001)

[Ma] S. Mardešić, Extension dimension of inverse limits, Glasnik Mat. 35(55) (2000), 339-354. MR.1812562 (2001i:54038)

[MR] S. Mardešić and L. Rubin, Cell-like mappings and nonmetrizable compacta of finite cohomological dimension, Trans. Amer. Math. Soc. 313 (1989), 53-79. MR0962284(90a:54095)

[MS1] S. Mardešić and J. Segal, Shape Theory, North-Holland, Amsterdam, 1982. MR0676973 (84b:55020)

[MS2] S. Mardešić and J. Segal, Stability of almost commutative inverse systems of compacta, Topology and its Appls. 31 (1989), 285-299. MR0997496 (90e:54018)

[Ru1] L. Rubin, Cell-like maps, dimension and cohomological dimension: a survey, Banach Center Publications 18 (1986), 371-376. MR0925877 (89a:55001)

[Ru2] L. Rubin, Cohomological dimension and approximate limits, Proc. Amer. Math. Soc. 125 (1997), 3125-3128. MR 1423333 (98g:55001)

[Ru3] L. Rubin, A stronger limit theorem in extension theory, Glasnik Mat. 36(56) (2001), 95103. MR1842828 (2002c:54031)

[RS] L. Rubin and P. Schapiro, Cell-like maps onto non-compact spaces of finite cohomological dimension, Topology and its Appls. 27 (1987), 221-244. MR0918533 (89b:55002)

[Sh] E. Shchepin, Arithmetic of dimension theory, Russian Math. Surv. 53 (1998), 975-1069. MR.1691185 (2002a:55002)

[Sp] E. Spanier, Algebraic Topology, McGraw-Hill, New York, 1966. MR0210112 (35:1007)

[Wa] J. Walsh, Dimension, cohomological dimension, and cell-like mappings, Shape Theory and Geometric Topology, Lecture Notes in Mathematics, volume 870, Springer Verlag, Berlin, 1981, pp. 105-118. MR0643526 (83a:57021)

Department of Mathematics, University of Oklahoma, 601 Elm Ave., Rm. 423, NorMAN, OKLAHOMa 73019

E-mail address: lrubin@ou.edu

Department of Mathematics, Langston University, Langston, Oklahoma 73050

E-mail address: pjschapiro@lunet.edu 